Published in final edited form as:

Cancer Immunol Res. 2019 April ; 7(4): 559-571. doi:10.1158/2326-6066.CIR-18-0637.

\title{
CD96 is an immune checkpoint that regulates CD8+ T-cell antitumor function
}

\author{
Deepak Mittal ${ }^{1,{ }^{*}}$, Ailin Lepletier ${ }^{1,{ }^{*}}$, Jason Madore ${ }^{1}$, Amelia Roman Aguilera ${ }^{1}$, Kimberley \\ Stannard ${ }^{1}$, Stephen J. Blake ${ }^{2}$, Vicki L. J. Whitehall ${ }^{3,4}$, Cheng Liu ${ }^{3,4,5}$, Mark L. Bettington ${ }^{5}$, \\ Kazuyoshi Takeda ${ }^{6,7}$, Georgina V. Long ${ }^{8,9,10}$, Richard A. Scolyer ${ }^{8,10}$, Ruth Lan ${ }^{11}$, Nathan \\ Siemers $^{11}$, Alan Korman ${ }^{11}$, Michele W. L. Teng ${ }^{2}$, Robert Johnston ${ }^{11}$, William C Dougall ${ }^{1,}{ }^{*}$, \\ and Mark J. Smyth ${ }^{1, *}$ \\ ${ }^{1}$ Immunology in Cancer and Infection Laboratory, QIMR Berghofer Medical Research Institute, \\ Herston, Queensland, Australia. \\ ${ }^{2}$ Cancer Immunoregulation and Immunotherapy, QIMR Berghofer Medical Research Institute, \\ Herston, Queensland, Australia.
}

${ }^{3}$ The Conjoint Gastroenterology Laboratory, QIMR Berghofer Medical Research Institute, Herston, Queensland, Australia.

${ }^{4}$ Faculty of Medicine, The University of Queensland, Herston, Queensland, Australia.

${ }^{5}$ Envoi Specialist Pathologists, Kelvin Grove, Queensland, Australia.

${ }^{6}$ Division of Cell Biology, Biomedical Research Center, Juntendo University, Bunkyo-ku, Tokyo 113-8421, Japan.

${ }^{7}$ Department of Biofunctional Microbiota Graduate School of Medicine, Juntendo University, Bunkyo-ku, Tokyo 113-8421, Japan.

${ }^{8}$ Melanoma Institute Australia, The University of Sydney, Sydney, NSW, Australia.

${ }^{9}$ Royal Prince Alfred Hospital, Sydney, NSW, Australia.

${ }^{10}$ The University of Sydney, NSW, Australia.

${ }^{11}$ Bristol-Myers Squibb, USA.

\section{Abstract}

CD96 is a novel target for cancer immunotherapy shown to regulate NK cell effector function and metastasis. Here, we demonstrated that blocking CD96 suppressed primary tumor growth in a number of experimental mouse tumor models in a $\mathrm{CD}^{+} \mathrm{T}$ cell-dependent manner. DNAM-1/

Corresponding Author: Mark J. Smyth, Immunology in Cancer and Infection Laboratory, QIMR Berghofer Medical Research Institute, 300 Herston Road, Herston 4006, Australia. mark.smyth@qimrberghofer.edu.au.

These authors contributed equally to this work.

Conflicts of Interest: Mark Smyth (M.J.S.) has been supported by a scientific research agreement with Bristol Myers Squibb, Corvus Pharmaceuticals, Aduro Biotech and Tizona Therapuetics. M. J. S. is on the scientific advisory board at Tizona Therapeutics and Nektar Therapeutics. William Dougall (W. C. D.) has received a speaker's honorarium from Amgen and has been supported by a scientific research agreement with Bristol Myers Squibb. Alan Korman, Nathan Siemers, Ruth Lan, and Robert Johnston are employees of Bristol Myers Squibb. Georgina V. Long is an advisor for Merck Sharpe Dohme, Novartis, Myriad and NeraCare. The other authors have no conflicts of interest to declare. 
CD226, Batf3, IL12p35, and IFN $\gamma$ were also critical, and CD96-deficient CD8 ${ }^{+} \mathrm{T}$ cells promoted greater tumor control than CD96-sufficient $\mathrm{CD}^{+} \mathrm{T}$ cells. The antitumor activity of anti-CD96 therapy was independent of Fc-mediated effector function and was more effective in dual combination with blockade of a number of immune checkpoints including PD-1, PD-L1, TIGIT, and CTLA-4. We consistently observed co-expression of PD-1 with CD96 on CD8 ${ }^{+} \mathrm{T}$ lymphocytes in tumor-infiltrating leukocytes both in mouse and human cancers using mRNA analysis, flow cytometry, and multiplex IHF. The combination of anti-CD96 with anti-PD-1 increased the percentage of IFN $\gamma$-expressing $\mathrm{CD} 8^{+} \mathrm{T}$ lymphocytes. Addition of anti-CD96 to antiPD1 and anti-TIGIT resulted in superior antitumor responses, regardless of the ability of the antiTIGIT isotype to engage FcR. The optimal triple combination was also dependent upon $\mathrm{CD} 8^{+} \mathrm{T}$ cells and IFN $\gamma$. Overall these data demonstrate that CD96 is an immune checkpoint on $\mathrm{CD} 8^{+} \mathrm{T}$ cells and that blocking CD96 in combination with other immune checkpoint inhibitors is a strategy to enhance T-cell activity and suppress tumor growth.

\section{Introduction}

Tumor antigen-specific $\mathrm{CD} 8^{+} \mathrm{T}$ cells become dysfunctional in the tumor microenvironment (TME), compromising their ability to proliferate and reducing effector function such as cytokine production and cytotoxicity. Therapeutic strategies to evoke antitumor immunity are largely aimed at reversing these immunosuppressive pathways. Antibody blockade of Tcell co-inhibitory receptors CTLA-4 and PD-1 or the immunosuppressive ligand PD-L1 has achieved impressive overall response rates in some cancer patients, in part, by reactivating tumor-specific $\mathrm{CD} 8^{+} \mathrm{T}$ cells (1). However, additional immunosuppressive signals originate from diverse sources in the TME, potentially circumventing PD-1/PD-L1 pathways and limiting the population of cancer patients who respond to current immunotherapies (2). The identification of additional immune suppressive ligands and the co-expression of additional co-inhibitory receptors on chronically activated $\mathrm{T}$ cells suggest that combined blockade of co-inhibitory receptors may improve response rates in cancer patients.

Certain proteins of the nectin and nectin-like (Necl) family, including CD155 and CD112, have emerged as candidate immune suppressive ligands which may circumvent immune reactivation after PD-1/PD-L1 blockade. These ligands can both activate lymphocyte function via interaction with the costimulatory Ig superfamily member DNAM-1/CD226 and, conversely, inhibit cell function through interaction with other Ig superfamily members, TIGIT and CD96 (reviewed (3)). We have demonstrated that CD155 is expressed on tumor cells and tumor-infiltrating myeloid cells in both human and mouse tumors and can impair antitumor T lymphocytes and NK cell function via interaction with TIGIT and CD96 (4). Importantly, the increased antitumor immunity upon blockade of PD-1 or PD-1 and CTLA-4 is more effective in settings in which CD155 is limiting (4), suggesting a mechanistic rationale for co-targeting PD-L1 and CD155 function. Blockade of the co-inhibitor receptors for CD155, TIGIT, and/or CD96 is one rational therapeutic approach for optimizing antitumor immunity.

Blockade of TIGIT in combination with anti-PD-L1 improves T-cell responses to tumors via an intrinsic effect on $\mathrm{CD} 8^{+}$T-effector cells leading to an increased production of IFN $\gamma$ and 
TNFa (5). TIGIT is also enriched on tumor-infiltrating T-regulatory cells (Tregs) compared to peripheral Tregs, and TIGIT expression on Tregs suppresses antitumor immunity (6). The expression pattern of CD96 is broadly similar between mice and humans, and CD96 is present on a proportion of T-effector and Tregs, NK cells, and NKT cells. CD96 expression is generally low or absent in tissues without lymphocyte infiltrate (reviewed in (3)). Earlier investigations of CD96 function have focused on an observed inhibitory function for CD96 on NK cells in anti-cancer immunity. For instance, the abrogation of lung metastases in a range of spontaneous and experimental models observed in $\mathrm{CD}^{-/-}$mice or upon CD96/ CD155 blockade with monoclonal antibody treatment was due to NK cell function, IFN $\gamma$, and effectively counterbalanced by the action of CD226 $(7,8)$.

We have confirmed CD96 expression in human $\mathrm{CD} 4^{+}$and $\mathrm{CD} 8^{+} \mathrm{T}$ cells and showed that CD96 mRNA expression was correlated with T-cell markers in primary and metastatic human tumors (9). However, T-cell function for CD96 in antitumor immunity remains undefined. Here, we showed that co-expression of CD96 with TIGIT and/or PD-1 in mouse and human tumor-infiltrating lymphocytes (TILs) and that using antibodies which selectively block CD155/CD96 interaction alone and in combination with anti-PD-1/PD-L1 regulates $\mathrm{T}$ cell-mediated tumor control.

\section{Materials and Methods}

\section{Mice}

C57BL/6 and BALB/c wild-type (WT) mice were purchased from the Walter and Eliza Hall Institute for Medical Research and bred in-house. C57BL/6.Rag2 ${ }^{-/} \mathrm{\gamma c}^{-/-}, \mathrm{C} 57 \mathrm{BL} /$ 6.Batf3 ${ }^{-1-}$, BALB/c.Batf3 ${ }^{-/}$, and C57BL/6 IL $12 p 35^{-1-}$ mice were maintained at QIMR Berghofer Medical Research Institute as previously described (7,10). C57BL/6 Tigit $^{-1}$ mice were kindly provided by Bristol Myers Squibb. C57BL/6.Cd96 ${ }^{-1-}$ and $C d 226^{-1-}$ mice were obtained from Dr Marco Colonna (Washington University School of Medicine, St Louis, MO, USA) and have already been described (7). All mice were bred and maintained at the QIMR Berghofer Medical Research Institute and used when more than 6 weeks of age. All experiments were approved by the QIMR Berghofer Medical Research Institute Animal Ethics Committee.

\section{Cell Culture}

B16F10 melanoma (ATCC, 2007), MCA1956 fibrosarcoma (Robert Schreiber, Washington University School of Medicine, St Louis, MO, USA, 2013), and CT26 colon carcinoma (Peter MacCallum Cancer Centre, 2012) were maintained for no more than two weeks culture, injected, and monitored as previously described $(11,12)$. All these cell lines express CD155 as previously described (4). Briefly, B16F10 cells were cultured in DMEM medium supplemented with 10\% heat-inactivated fetal calf serum (Thermo Scientific), 1X glutamax, penicillin $(50 \mathrm{U} / \mathrm{mL})$, streptomycin $(100 \mu \mathrm{g} / \mathrm{mL})$, and 10mM HEPES (Sigma-Aldrich), whereas other cell lines were cultured in RPMI supplemented with $10 \%$ heat-inactivated fetal calf serum, 1X glutamax, penicillin (50 U/mL), streptomycin $(100 \mu \mathrm{g} / \mathrm{mL}), 1 \mathrm{mM}$ sodium pyruvate from Gibco-Life Technologies and $10 \mathrm{mM}$ HEPES in $5 \% \mathrm{CO}_{2}$. All mouse 
tumor cell lines were tested for mycoplasma using the Lonza Mycoalert Mycoplasma

Detection Kit, but cell line authentication was not routinely performed.

\section{Tumor growth and treatments}

Tumor cells, B16F10 $\left(1 \times 10^{5}\right)$, CT26 $\left(2 \times 10^{5}\right)$ and MCA1956 $\left(1 \times 10^{6}\right)$, were injected subcutaneously into the left flank of 6-20 weeks old C57BL/6 or BALB/c wild-type (WT) mice or various C57BL/6 gene-targeted mice. Mice were treated with i.p injections of 250 $\mu \mathrm{g}$ of anti-CD96 (3.3, rat IgG1, BioXCell), anti-TIGIT (4B1, mouse IgG1 D265A, or IgG2a, Bristol Myers Squibb), anti-PD-1 (RMP1-14, BioXCell), anti-PD-L1 (10F.9G2, rat IgG2b, BioXCell), anti-CTLA-4 (UC10-4H10, hamster Ig), or control IgG (1-1, rat IgG1, BioXCell) in schedules as indicated. In some experiments, anti-CD8 $\beta$ (53.5.8, $100 \mu \mathrm{g})$, antiDNAM-1 $(480.1,250 \mu \mathrm{g})$, anti-asGM1 $(50 \mu \mathrm{g})$, or anti-IFN $\gamma(\mathrm{H} 22,250 \mu \mathrm{g})$ from BioXCell (West Lebanon, NH) were injected i.p. immediately prior and during immunotherapy treatment as indicated. Tumor size was measured every two to four days a week with a digital caliper as the product of two perpendicular diameters. Survival was measured by plotting the last day of ethical tumor size measurement as the time of sacrifice.

\section{Adoptive transfer of $\mathrm{CD8}^{+} \mathrm{T}$ cells}

Splenocytes from WT and CD96 ${ }^{-/}$mice were stained with TCR $\beta$-PerCP-Cy5.5 (H57-597), CD8-BV711 (53.6.7), and zombie aqua and CD8 ${ }^{+} \mathrm{T}$ cells (live $\mathrm{TCR} \beta^{+} \mathrm{CD} 8^{+}$) were sorted on

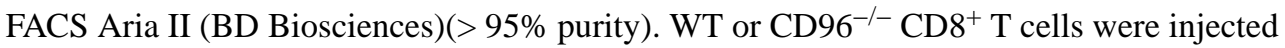
intravenously into $\mathrm{Rag} 2^{-/-} \gamma \mathrm{c}^{-/-}$mice. After 10 days, blood was collected to check the equivalent reconstitution of $\mathrm{CD}^{+} \mathrm{T}$ cells by flow cytometry. B16F10 $\left(1 \times 10^{5}\right)$ melanoma cells were injected subcutaneously into C57BL/6 $\mathrm{Rag}^{-/-} \gamma^{-/-}$mice and mice were monitored for the tumor growth.

\section{MCA-Induced Fibrosarcoma}

Groups of 10-15 C57BL/6 WT male mice were inoculated s.c. in the hind flank with $300 \mu \mathrm{g}$ of methylcholanthrene (MCA) (Sigma-Aldrich) in $0.1 \mathrm{~mL}$ of corn oil as described previously (13). Mice were treated with cIg (1-1), anti-CD96 (3.3), anti-PD-1 (RMP1-14), anti-TIGIT (4B1, IgG2a), anti-TIGIT (4B1, D265A) or their combinations (100 $\mu$ g each i.p., twice/week) for 6 weeks from the second palpable tumor measurement $\left(0.1-0.4 \mathrm{~cm}^{2}\right.$, days 84-147 relative to MCA inoculation). Mice were then monitored for fibrosarcoma development over 250 days, with measurements made with a caliper square as the product of two perpendicular diameters $\left(\mathrm{cm}^{2}\right)$. Data were recorded as tumor size in $\mathrm{cm}^{2}$ of individual mice, or tumor growth rate $\left(\mathrm{mm}^{2} /\right.$ day) relative to treatment initiation.

\section{Flow cytometry}

Tumors were cut into small pieces and digested in digestion medium containing RPMI with collagenase II ( $1 \mathrm{mg} / \mathrm{mL})$ and DNAse $(20 \mu \mathrm{g} / \mathrm{mL})$ for 45 minutes. Tumors and spleen samples were filtered through $70 \mu \mathrm{m}$ filter, washed in PBS, and red blood cells were lysed by ACK buffer (0.15 $\mathrm{M} \mathrm{NH}_{4} \mathrm{Cl}, 10 \mathrm{mM} \mathrm{KHCO}_{3}$, EDTA $0.1 \mathrm{mM}, \mathrm{pH}$ 7.2-7.4). Single-cell suspensions were incubated for 15 minutes in Fc blocking buffer $(2.4 \mathrm{G} 2$ antibody in $2 \%$ FBS and $1 \mathrm{mM}$ EDTA in PBS) before staining with following antibodies in $2 \%$ FCS PBS: 
anti-mouse CD45.2-APCcy7 (104), TCR $\beta$-PerCP-Cy5.5 (H57-597), CD4-BV605 (RM4-5), CD8-BV711 (53.6.7), CD96-PE (3.3), TIGIT-APC (Vstm3), PD-1-FITC (J43), and zombie yellow or zombie aqua for live-dead stain. All mAbs were purchased either from Biolegend (San Diego, CA) or eBioscience (San Diego, CA). For AH1 (gp70) tetramer staining, H-2Ld MuLV gp70 Tetramer-SPSYVYHQF-PE or -APC was obtained from NIH Tetramer Core Facility, and samples were incubated with $\mathrm{AH} 1$ tetramer in complete RPMI medium at $37^{\circ} \mathrm{C}$ in 5\% $\mathrm{CO}_{2}$ incubator for 2 hours and then washed with PBS before staining with other antibodies. For intracellular cytokine staining (ICS), samples were either stimulated with gp70 peptide (sequence SPSYVYHQF) in the presence of brefeldin A (1000x) or with Cell Stimulation Cocktail (plus protein transport inhibitors) (1000x) (eBioscience) for 4 hours in complete RPMI medium at $37^{\circ} \mathrm{C}$ before staining with cell surface antibodies as described above. Samples were fixed and permeabilized using fixation permeabilization buffer from eBioscience for 20 minutes and frozen at $-80^{\circ} \mathrm{C}$. Samples were thawed for 20 minutes, washed, and then stained with anti-mouse FoxP3-efluor450 (FJK-16S), IFN $\gamma$-APC (XMG1.2), TNFa-BV605 (Mab11). To determine absolute counts in samples, liquidcounting beads (BD Biosciences) were added directly before samples were run on a flow cytometer. Samples were acquired on LSR Fortessa IV Flow Cytometer (BD Biosciences) and data was analyzed on FlowJo V10 (Treestar).

\section{mRNA analyses}

For analysis of melanoma single cell RNA-seq, processed from Tirosh et al (14) were obtained from the Broad Single Cell Portal (https://portals.broadinstitute.org/single_cell). Visualization tools and generation of scatter plots of transcript levels from a single-cell RNA-seq data study of melanoma patients were done as described (15). RNA-seq data from the TCGA project was obtained from the UCSC Cancer Genomics Hub. To determine the correlation between expression of the CD96 with other genes of interest, RNA-sequencing data normalized using the edgeR package. Pearson's rank correlation was estimated on the normalized counts.

\section{Multiplexed immunohistofluorescence (mIHF), image processing, and analysis of human tumors}

Archival colorectal cancer (CRC) samples were obtained from Envoi Specialist Pathologists and archival melanoma samples were obtained from Melanoma Institute Australia. The study protocols were approved by the QIMR Berghofer Human Research Ethics Committee (P1298, P2125). Microsatellite instability (MSI) status was assigned to CRC samples based on immunohistochemical absence of the mismatch repair protein MLH1, which serves as a surrogate for MSI. A multispectral fluorescence imaging panel that examined CD8, PD-1, and CD96 with either SOX10 for melanoma or SATB2 for colorectal cancer was used to quantitate expression of CD96 and PD- 1 on $\mathrm{CD} 8^{+} \mathrm{T}$ cells. DAPI was used as a nuclear stain. Briefly, specimens were sectioned at $4 \mu \mathrm{m}$ onto superfrost+ microscope slides and stored under vacuum until mIHF was performed. Heat-induced antigen retrieval with EDTA target retrieval buffer (DAKO) was performed using a microwave and staining was run on an automated tissue stainer (DAKO). Primary antibodies were visualized using the OPAL multiplex TSA detection system (PerkinElmer) as per the manufacturer's instructions with heating for 20 minutes at $100^{\circ} \mathrm{C}$ using EDTA buffer between sequential staining rounds to 
strip prior bound antibody/HRP complexes. Primary antibodies, working dilutions, secondary detection HRP antibodies, and OPAL TSA dyes are listed in Supplementary Table $\mathrm{S} 1$. Fluorescence-stained slides were scanned using a Vectra imaging system (PerkinElmer). Whole slide scanning was done at $4 \mathrm{x}$ magnification using mixed fluorescence, and regions for 20x multispectral imaging were selected based on tumor marker and CD8 signals. For each sample, two to five $20 \times$ multispectral imaging regions were selected to represent the tumor immune microenvironment. Multispectral images were spectrally unmixed followed by tissue and cell segmentation using Inform analysis software (PerkinElmer; v2.2.1). Nuclear expression of SATB2 or SOX10 by tumor cells was used to segment tumor and stroma tissue regions. Merged data files were pre-processed and fluorescence thresholds were set using Spotfire image mapping tools (Tibco Spotfire Analyst; v7.6.1) followed by segmented cell counting using Spotfire.

\section{Statistical Analysis}

Statistical analysis was achieved using Graphpad Prism Software. Data were considered to be statistically significant when the $\mathrm{p}$ value was equal to or less than 0.05 . Data were compared using a Mann-Whitney $U$ test. Differences in survival were evaluated using a Mantel-Cox test.

\section{Results}

\section{Tumor growth suppression by anti-CD96 requires $\mathrm{CD} 8^{+} \mathrm{T}$ cells and IFN $\gamma$}

Although targeting of the CD96 immune checkpoint on NK cells has been validated as an approach to reduce both experimental and spontaneous metastasis (7), the role of CD96 on $\mathrm{CD}^{+} \mathrm{T}$ cells in primary tumor immunity is largely unknown. Treatment of mouse CT26 colon carcinoma, B16F10 melanoma, and MCA1956 fibrosarcoma with anti-CD96 minimally reduced tumor growth (Fig. 1A-C). The antitumor activity of anti-CD96 therapy was independent of Fc-mediated effector function in the MCA1956 tumor model (Supplementary Fig. S1A). To determine the mechanism of action of anti-CD96, depleting antibodies for either NK cells or $\mathrm{CD}^{+} \mathrm{T}$ cells and neutralizing antibodies to IFN- $\gamma$ were employed from the commencement of anti-CD96 therapy. In the MCA1956 fibrosarcoma model, depletion of $\mathrm{CD}^{+} \mathrm{T}$ cells and neutralization of IFN $\gamma$ completely abolished the efficacy of anti-CD96 therapy, whereas NK cell depletion had no significant impact (Fig. 1D-E). $\mathrm{CD}^{+} \mathrm{T}$ cells also express perforin as a critical effector molecule. However, antiCD96 therapeutic benefit was retained in perforin-deficient mice (Fig. 1E). The single agent anti-CD96 efficacy observed in the MCA1956 fibrosarcoma (Fig. 1C) was reversed by the addition of blocking anti-CD226/DNAM-1 in WT mice or absent in DNAM-1 ${ }^{-/}$mice (Fig. $1 F)$. Depletion of $\mathrm{CD}^{+} \mathrm{T}$ cells also significantly reduced the efficacy of anti-CD96 therapy in the B16F10 melanoma tumor model (Supplementary Fig. S1B). To define whether CD96 suppressed antitumor $\mathrm{CD}^{+} \mathrm{T}$ cells directly or indirectly, we utilized adoptive transfer of WT or CD96-deficient (CD96 ${ }^{-/-}$) T cells into immunodeficient $\mathrm{Rag}^{-/-} \mathrm{\gamma c}^{-/-}$mice bearing B16F10 tumors. Superior tumor control was observed when CD96-deficient CD8 ${ }^{+}$T cells were adoptively transferred compared with transfer of WT CD96-expressing CD8 ${ }^{+} \mathrm{T}$ cells (Supplementary Fig. S1C), suggesting that CD96 expression on CD8 ${ }^{+}$T cells directly limits their antitumor function. Overall these results indicated that anti-CD96 therapy minimally 
controlled tumor burden as a monotherapy and required CD8 ${ }^{+} \mathrm{T}$ cells, CD226/DNAM-1, and IFN $\gamma$, but not NK cells and perforin.

\section{Anti-CD96 therapy requires Batf3+ cross-presenting dendritic cells and IL12}

Batf $^{+}$cross-presenting dendritic cells (DCs) are major producers of IL12 and are critical regulators of tumor growth and metastasis $(10,16,17)$. Immunotherapies such as anti-PD-L1 and the combination of anti-PD-1 and anti-CD137 require Batf3-dependent DCs $(18,19)$. The antitumor efficacy of anti-CD96 therapy was completely lost in Batf3-deficient mice and in IL12p35-deficient mice (Fig. 2A-D), suggesting that the mechanism of anti-CD96 is dependent on Batf $3^{+}$DCs, possibly through their IL12 secretion.

\section{CD96 is co-expressed with PD-1 and TIGIT on human and mouse TILs}

Given the obligate role for $\mathrm{CD} 8^{+} \mathrm{T}$ cells in the antitumor efficacy of anti-CD96 treatment, we evaluated the expression of CD96 on mouse and human tumor-infiltrating lymphocytes (TILs). Analysis of CT26 colon carcinoma tumors indicated the CD96 expression was predominantly on $\mathrm{CD}^{+} \mathrm{T}$ cells and was observed less frequently on $\mathrm{CD} 4{ }^{+} \mathrm{FoxP} 3^{-}$and $\mathrm{CD}^{+}{ }^{+} \mathrm{FoxP}^{+}{ }^{+} \mathrm{T}$ cells (Supplementary Fig. S2A-C). On CD8 ${ }^{+} \mathrm{T}$ cells, CD96 was coexpressed with the other inhibitory receptors PD-1 or TIGIT ( $20 \% \mathrm{CD} 8^{+} \mathrm{T}$ cells) and sometimes observed as $\mathrm{CD} 96^{+} \mathrm{TIGIT}^{+} \mathrm{PD}-1^{+}$cells (4.8\% of $\mathrm{CD} 8^{+} \mathrm{T}$ cells). CD96, PD-1, and TIGIT expression on antigen-specific gp $70^{+} \mathrm{CD} 8^{+}$TILs was relatively higher than on gp70negative CD8 ${ }^{+}$TILs in CT26 tumors (Supplementary Fig. S3A).

Evaluation of gene expression data from the Cancer Genome Atlas (TCGA) demonstrated that CD96 mRNA was correlated with T-cell markers, such as CD3E, CD4, and CD8A, across most tumor types represented (9). Here, we showed that expression of CD96 was also correlated with expression of PD-1, a marker of T-cell dysfunction upon chronic antigen exposure. CD96 expression was also correlated with other candidate co-inhibitory Ig superfamily receptors TIGIT and PVRIG/CD112R and its complementary co-stimulatory receptor, CD226/DNAM-1 Supplementary Fig. S3B) across most tumor types tested. Analysis of each of the 32 tumor types available from TCGA indicated that the correlation of CD96 with these markers was consistently observed, with correlations between CD96 and TIGIT observed (22/32 tumor types demonstrating a Pearson's correlation coefficient > 0.75). Correlations between CD96 and PD-1 were also observed (13/32 tumor types demonstrating a Pearson's correlation coefficient > 0.75) (Supplementary Fig. S3B).

Next, we analyzed the expression of CD96 and its correlation with relevant markers in human melanoma single-cell RNA-seq data from Tirosh et al. (14). Gene expression was tested selectively in the $\mathrm{CD}_{4}{ }^{+} \mathrm{CD} 3 \mathrm{E}^{+} \mathrm{CD} 8 \mathrm{~A}^{+} \mathrm{CD} 19^{-} \mathrm{ITGAM}^{-}$population and stratified according to PDCD $1^{\text {low }}$ versus PDCD $1^{\text {high }}$ expression. CD96 mRNA was co-expressed with CD8A in melanoma TILs (39\%; Supplementary Fig. S4), and this expression was detected in both PDCD $1^{\text {low }}(15 \%)$ and PDCD $1^{\text {high }}(46 \%) \mathrm{CD} 8^{+} \mathrm{T}$ cells. Co-expression of CD96 with TIGIT mRNA was also observed in CD8 ${ }^{+}$melanoma TILs (54\%), and again, this coexpression was observed in both PDCD $1^{\text {low }}$ and PDCD $1^{\text {high }} \mathrm{CD} 8^{+} \mathrm{T}$ cells (Supplementary Fig. S4). 
To confirm protein expression of CD96 by tumor-infiltrating T cells, we evaluated CD96 expression in colorectal cancer by multiplexed immunohistochemistry (mIHF) (Fig. 3A). A cohort of 7 microsatellite unstable human primary colorectal cancers were selected (MSICRCs) and had higher immune cell infiltration and were amenable to immunotherapy checkpoint blockade (20). The number of $\mathrm{CD} 8^{+} \mathrm{T}$ cells per 20x high-power field (HPF) were counted. Consistent with the mRNA analysis, $\mathrm{CD} 96^{+}$lymphocytes were observed in CRCs, with a subpopulation staining $\mathrm{CD} 96$ amongst $\mathrm{PD} 1{ }^{+} \mathrm{CD} 8^{+} \mathrm{T}$ cells (Fig. 3B). $\mathrm{PD}-1^{+} \mathrm{CD} 8^{+} \mathrm{T}$ cells were significantly enriched within the tumor parenchyma vs. tumor stroma (Fig. 3C). Although the overall frequency of CD $96^{+} \mathrm{PD}-1^{+} \mathrm{CD} 8^{+} \mathrm{T}$ cells (median $=0.4$ cells $/ \mathrm{HPF}$, range $\left(0.0\right.$ - 22.4)) was less than $\mathrm{CD}^{-} 6^{-} \mathrm{PD}-1^{+}($median $=16.4$ cells $/ \mathrm{HPF}$, (range $\left.2.0-90.4)\right)$, a trend towards increased CD $96^{+} \mathrm{PD}-1^{+} \mathrm{CD} 8^{+} \mathrm{T}$ cells was observed in the tumor parenchyma of patients with $\mathrm{CD}^{+} \mathrm{T}$-cell infiltrated tumors. Unfortunately, many efforts to detect TIGIT by IHF were unsuccessful. Flow cytometry analysis of 8 archival CRC samples revealed that CD96 was expressed on most PD- $1^{\text {high }}$ and PD- $1^{\text {low }} \mathrm{CD} 8^{+} \mathrm{T}$ cells (Supplementary Fig. S5). TIGIT was expressed less often than CD96 but did trend for expression on PD- $1^{\text {high }} \mathrm{CD} 8^{+} \mathrm{T}$ cells.

Similar results were observed by multiplex IHF of a cohort of 9 human metastatic melanoma samples (Fig. 4A-C). In melanoma CD $96^{+} \mathrm{PD}-1^{+} \mathrm{CD} 8^{+} \mathrm{T}$ cells $($ median $=31$ cells $/ \mathrm{HPF}$, (range 4.5 -203.5)) were more frequent than was observed for CRC but in both tumor types, the cells were found at higher numbers in the tumor parenchyma. For melanoma, we identified a population of $\mathrm{CD} 96^{+} \mathrm{PD}-1^{-} \mathrm{CD} 8^{+} \mathrm{T}$ cells (median $=17$ cells $/ \mathrm{HPF}$, (range $0.5-$ 287.5)) that was not present in CRC. There was no difference in distribution of $\mathrm{CD} 96^{+} \mathrm{PD}-1^{-} \mathrm{CD} 8^{+} \mathrm{T}$ cells between tumor stroma and parenchyma. These data suggested that tumor-infiltrating CD8 ${ }^{+} \mathrm{T}$ cells can co-express CD96 and PD-1 and that these cells might localize to the tumor parenchyma in CRC and melanoma.

\section{Anti-CD96 therapy enhances the efficacy of immune checkpoint blockade}

Given that CD96 is frequently co-expressed with PD-1 and TIGIT in both mouse and human intratumor $\mathrm{CD} 8^{+} \mathrm{T}$ cells, we hypothesized that the combination of anti-CD96 with antibodies blocking the immune checkpoints PD1/PD-L1 and TIGIT might enhance antitumor responses. We observed that concurrent treatment with anti-CD96 enhanced the efficacy of anti-PD-1, anti-PD-L1, and anti-CTLA4 in the CT26 colon carcinoma model (Fig. 5A-B). The combination of anti-CD96 with anti-TIGIT (IgG2a) decreased the growth of MCA1956 fibrosarcoma tumors and increased the number of tumor-free mice (Fig. 5CD). These results suggested that anti-CD96 enhanced the antitumor efficacy of multiple immune checkpoint inhibitors. The mechanism of combination anti-CD96 with anti-TIGIT was dependent on $\mathrm{CD}^{+} \mathrm{T}$ cells but not on NK cells, as the therapeutic efficacy of the antiCD96/anti-TIGIT combination was lost in CD8 ${ }^{+} \mathrm{T}$ cell-depleted WT mice but retained in mice where NK cells were depleted with anti-asGM1 (Supplementary Fig. S6).

Given the superior activity of the anti-PD-1/CD96 dual combination in the CT26 tumor model, we next assessed the functional consequences of CD96 and PD-1 inhibition on TILs. Here, we demonstrated an increased frequency of intratumor $\mathrm{CD} 8^{+} \mathrm{T}$ cells, but not $\mathrm{CD} 4^{+} \mathrm{T}$ effector cells or Tregs, in mice treated with the combination of anti-PD-1 and anti-CD96 
(Fig. 6 and Supplementary Fig. S7). Among CD8 ${ }^{+} \mathrm{T}$ cells, an increase in the frequency of IFN $\gamma^{+}$and IFN $\gamma^{+} \mathrm{TNFa}^{+} \mathrm{CD} 8^{+} \mathrm{T}$ cells was observed after treatment with the combination of anti-PD-1 and anti-CD96 compared to the control Ig or anti-CD96 or anti-PD-1 monotherapies (Fig. 6 and Supplementary Fig. S7). These data suggested that blockade of CD96 and PD-1 selectively and synergistically enhanced the effector function of tumorinfiltrating $\mathrm{CD} 8^{+} \mathrm{T}$ cells, consistent with the co-expression of these receptors on TILs and the requirement for $\mathrm{CD}^{+} \mathrm{T}$ cells in anti-CD96/PD-1 treatment efficacy.

\section{Anti-CD96 enhances TIGIT and PD-1 blockade in a triple combination therapy}

To potentially improve the therapeutic benefit of immune checkpoint blockade (ICB), we tested anti-PD-1/CD96/TIGIT in a triple combination therapy. The triple combination of anti-PD-1/CD96/TIGIT was superior in reducing the tumor growth and improving the survival of mice in B16F10 melanoma and CT26 colon carcinoma compared to any monotherapy or dual combination therapy (Fig. 7A-B and Supplementary Fig. S8A-B). The antitumor response achieved with each dual combination treatment was significantly greater than monotherapy efficacies, with the dual combination of anti-PD-1 and anti-TIGIT (mouse IgG2a) having the best response (Fig. 7A-B).

TIGIT is expressed on intratumor Tregs (Fig. 3A), and TIGIT expression on Tregs has a reportedly prominent role in suppressing antitumor immunity compared to TIGIT expression on $\mathrm{CD}^{+} \mathrm{T}$ cells (6). Therefore, we questioned whether the enhanced antitumor efficacy of anti-TIGIT (IgG2a) with anti-PD-1 (IgG2a) or anti-CD96 (IgG1) in dual or triple combination therapy involved Fc receptor-mediated depletion of Tregs. To test this, we used anti-mouse TIGIT with different $\mathrm{Fc}$ isotypes (IgG1 vs. IgG2a vs. Fc-mutant D265A) in dual or triple combination therapy with anti-CD96 and anti-PD-1. Anti-TIGIT IgG2a was superior to anti-TIGIT IgG1 either as a monotherapy or in combination with anti-CD96 in reducing B16F10 melanoma tumor growth (Fig. 7C). Anti-TIGIT IgG2a also had a greater antitumor efficacy than Fc-mutant anti-TIGIT D265A as a monotherapy or when combined with anti-CD96 in dual or anti-CD96 and anti-PD-1 in triple combination therapy (Fig. 7D, Supplementary Fig. S8C, Supplementary Fig. S9A). The mechanism of triple combination of anti-PD-1, anti-CD96, and anti-TIGIT (G2a) was dependent on CD8 ${ }^{+} \mathrm{T}$ cells and IFN $\gamma$ as depletion of $\mathrm{CD}^{+} \mathrm{T}$ cells and neutralization of IFN $\gamma$ almost completely abrogated the antitumor efficacy of triple therapy (Supplementary Figure 9B-C). The triple combination therapy of anti-PD-1/CD96/TIGIT(G2a) may require NK cells, in addition to CD8 ${ }^{+} \mathrm{T}$ cells, as the depletion of NK cells somewhat reduced the efficacy of the triple combination when used alone or in combination with $\mathrm{CD}^{+}$T-cell depletion (Supplementary Fig. S9B).

\section{Triple ICB therapy is effective against de novo carcinogen-induced tumors}

To test the efficacy of triple combination therapy in a model of established fibrosarcomas induced in WT mice by s.c. injection of $300 \mu \mathrm{g}$ MCA, mice were treated with anti-CD96, anti-TIGIT (G2a vs D265A), and anti-PD-1 or control IgG over a six-week period, either alone or as dual or triple combination therapy. Consistent with the results from the transplanted tumor models (Figs. 5 and 7), triple combination of anti-PD-1/CD96/TIGIT was superior at slowing tumor growth, resulting in more tumor-free mice (50\%) compared to either monotherapy or dual combination therapy (Fig. 8, Supplementary Fig. S10). The 
number of tumor-free mice in single, dual, or triple combination therapy was greater when mice were treated with $\mathrm{Fc}$ active anti-TIGIT IgG2a, than with Fc-mutant anti-TIGIT D265A [single anti-TIGIT: IgG2a 3/12 (25\%) vs D265A 1/15 (6.6\%); dual: anti-PD1/TIGIT: IgG2a 5/12 (41.6\%) vs D265A 3/16 (18.7\%); dual anti-CD96/TIGIT: IgG2a 3/12 (25\%) vs D265A 2/15 (13.3\%); and triple anti-PD1/CD96/TIGIT: IgG2a 6/12 (50\%) vs 8/24 (33.3\%). Overall these results suggest that the triple blockade of PD-1, CD96, and TIGIT was a superior therapeutic approach over the monotherapy or dual therapy combinations tested, and the Fc backbone of the anti-mouse TIGIT antibody plays a role in determining its antitumor efficacy.

\section{Discussion}

The receptors CD96, TIGIT, and CD226/DNAM-1 comprise a critical regulatory system for lymphocyte activity and antitumor immunity. CD96, TIGIT, and CD226/DNAM-1 share the same ligands CD112 and CD155, and engagement of CD226 activates NK cell and T-cell activity, whereas TIGIT and CD96 are thought to counterbalance CD226-dependent lymphocyte activation. Prior work has demonstrated that CD96 is an intrinsic inhibitory receptor on NK cells, as the genetic deletion of CD96 or blockade using CD96-specific mAbs enhances NK cell IFN $\gamma$ production and decreases spontaneous or experimental lung metastases $(7,8)$. Although it has been clearly demonstrated that TIGIT is an intrinsic T-cell inhibitory receptor and can suppress CD226 activation of T cells $(5,6,21-23)$, it has been unclear whether CD96 is intrinsically functional as an inhibitory receptor on T cells.

Here, we showed that CD96 was highly expressed in both human and mouse tumorinfiltrating $\mathrm{CD}^{+} \mathrm{T}$ cells and was observed along with other co-inhibitory receptors such as PD-1 and TIGIT, suggesting an association with increased dysfunction of CD8 $8^{+} \mathrm{T}$ cells. Pharmacologic blockade of CD96-CD155 interactions using CD96-specific mAbs significantly controlled subcutaneous tumor growth in multiple mouse models, via a mechanism that was independent of NK cells and, instead, required CD8 ${ }^{+} \mathrm{T}$ cells and IFN $\gamma$. The antitumor efficacy of anti-CD96 was dependent on the complementary co-stimulatory receptor CD226/DNAM-1, and blockade of CD96 enhanced the antitumor efficacy of antiPD-1/PD-L1, which correlated with an enhancement of tumor-infiltrating CD8 ${ }^{+}$T-cell effector function. These data validate CD96 as an inhibitory receptor which controls antitumor $\mathrm{CD}^{+}$effector functions and suggest that targeting CD96 may supplement the foundation strategy of PD-1/PD-L1 inhibition to improve therapeutic responses.

Blockade of CD96 not only enhanced PD-1/PD-L1 inhibition, but also the antitumor effect of anti-TIGIT, leading to increased tumor control and regression. Given that TIGIT can uniquely bind CD112, whereas both CD96 and TIGIT bind CD155, the superior combination efficacy of saturating concentrations of blocking TIGIT and CD96 antibodies cannot be explained by different ligand binding interactions of each inhibitory receptor. The superior combination efficacy of anti-CD96 plus anti-TIGIT was dependent on CD8 ${ }^{+} \mathrm{T}$ cells but independent of NK cells, suggesting that the T-cell function of these inhibitory receptors is non-redundant. 
The non-redundant mechanisms of CD96 and TIGIT blockade can be explained in multiple ways: 1) selective effects of each pathway on different cell types or distinct anatomic or tissue-specific compartments; 2) distinct functional response of each receptor on same cell; or 3) distinct temporal expression of the CD96 and TIGIT on T cells. Although CD96 and TIGIT are co-expressed on a sub-fraction of human and mouse tumor infiltrating T cells, including those marked by increased PD-1 expression, TIGIT expression is distinctly enriched in Tregs compared with CD96. Kurtulus et.al. show that TIGIT function in Teffector cells is not as critical as the immunosuppressive effect of TIGIT on Tregs in tumor control (6). Conversely, analysis of anti-CD96 monotherapy efficacy or the improved tumor control upon adoptive transfer of CD96 gene-targeted CD8 ${ }^{+}$cells (compared with transfer of $\mathrm{CD} 6^{+} \mathrm{CD}^{+}$cells) in this current study indicated a role for $\mathrm{CD} 96$ on dysfunctional Teffector cells, whereas evidence for CD96 function on Tregs in antitumor immunity is still lacking. To address the cell type-selective effects of TIGIT and CD96 mAbs, we utilized different $\mathrm{Fc}$ variants of the TIGIT mAb and showed the greatest antitumor efficacy when anti-TIGIT mIgG2a was used in combination with anti-CD96. We cannot exclude distinct functional activities of the two inhibitory receptors on $\mathrm{CD} 8^{+} \mathrm{T}$ effector cells as enhanced antitumor control was also observed with combination anti-CD96 plus Fc-mutated antiTIGIT mIgG1 D265A.

Optimal clinical efficacy can theoretically be achieved by combination immunotherapies targeting novel checkpoint receptors to improve tumor-specific immune responses. We demonstrated that the checkpoint inhibitor CD96 was expressed in parallel with PD-1 and TIGIT in human and mouse tumor-infiltrating $\mathrm{CD} 8^{+} \mathrm{T}$ cells. Indeed, anti-CD96 treatment enhanced tumor control in combination with anti-TIGIT plus anti-PD-1, not only in the transplanted CT26 model, but also the de novo MCA-induced fibrosarcoma model.

Altogether, these data suggest that CD96, TIGIT, and PD-1 represent non-redundant mechanisms of tumor-induced immune suppression and T-cell dysfunction and can be cotargeted to achieve superior antitumor responses.

We have demonstrated that anti-CD96 enhances the antitumor efficacy of multiple methods of ICB and that CD96 is co-expressed with PD- 1 in human tumor-infiltrating CD8 ${ }^{+}$cells. These findings suggest that a therapeutic strategy for co-targeting these receptors in human cancers is tractable. The current study regarding CD96 and TIGIT expression and function and the prior observation that limiting CD155 will enhance anti-PD-1 responsiveness in a preclinical setting (4) together suggest that pre-treatment expression of CD155 and/or CD96 and TIGIT may define a patient population refractory to PD-1/PD-L1 monotherapy. Correlation of immunotherapy response and baseline CD155 in tumors or baseline and/or on-treatment CD96 and TIGIT expression in TILs may provide insight into the clinical application of these approaches.

However, given that functional response to anti-TIGIT or anti-CD96 requires CD226 function $\left((5,24)\right.$ and this study, respectively) any strategy to restore $\mathrm{CD} 8^{+} \mathrm{T}$ lymphocyte reactivity against cancer by inhibiting ligand binding to TIGIT or CD96 should consider the contribution of CD226. Although we demonstrated that CD96 and CD226 transcripts were correlated in tumor tissue, protein co-expression on tumor-infiltrating $\mathrm{CD} 8^{+} \mathrm{T}$ cells remains to be demonstrated, and there is evidence that CD226/DNAM-1 expression may be down- 
modulated upon chronic antigen stimulation. For instance, downregulation of CD226 has been observed on virus-specific PD $1{ }^{+} \mathrm{Lag} 3^{+} \mathrm{CD} 8^{+} \mathrm{T}$ cells in both HIV and LCMV chronic infections (25) and, in the cancer setting, CD226 is reduced in chronically-stimulated NK cells in a mouse multiple myeloma model (26) and CD8 $8^{+}$TILs from melanoma patients (22). Conversely, increased expression of CD226/DNAM-1 on TILs corresponded with a greater response to anti-PD-1/PD-L1 or anti-CTLA-4 therapies (4). A lower ratio of activating (CD226): inhibitory (CD96, TIGIT) receptors will potentially limit lymphocyte responses, and these ratios might be individually regulated in different cell types. The expression of CD96 or TIGIT in patient tumor-infiltrating CD8 ${ }^{+} \mathrm{T}$ cells may not be sufficient to predict response to anti-TIGIT or anti-CD96 and functional expression of CD226/DNAM-1 should also be considered.

The strategy of targeting novel checkpoint receptors in combination may simultaneously block non-redundant immunosuppressive mechanisms to further increase clinical efficacy, as exemplified by the increased clinical response in patients with advanced melanoma receiving concurrent blockade of CTLA-4 and PD-1 compared with patients receiving monotherapy (27). However, this combination strategy may come at the cost of increased frequency and severity of immune-related adverse effects (irAEs), as has been observed upon combination treatment with anti-PD-1 with anti-CTLA-4 (28). We have generated gene-targeted mice that were double deficient for either PD-1 and CD96 or TIGIT and CD96 and observed no long-term immune-related toxicities or autoimmunity (29). These data suggest that cotargeting these pathways can enhance tumor efficacy without inducing serious immunerelated toxicities.

In summary, our data revealed that CD96 is expressed on infiltrating CD8 ${ }^{+} \mathrm{T}$ cells in mouse and human tumors where it functions as an intrinsic inhibitory receptor and that blockade of CD96 either as a monotherapy or in combination with blockade of another Ig superfamily member, TIGIT, or checkpoint inhibitors leads to enhanced antitumor immunity. Along with prior data indicating an inhibitory role for CD96 on NK cells, our study provides the rationale for clinical evaluation of CD96 antagonists targeting multiple immune compartments as a strategy for cancer immunotherapy.

\section{Supplementary Material}

Refer to Web version on PubMed Central for supplementary material.

\section{Acknowledgements}

The authors wish to thank Liam Town and Kate Elder for genotyping and maintenance and care of the mice used in this study. We would also like to thank the Bristol Myers Squibb team for support in producing the anti-TIGIT used in this project. The authors would like to acknowledge the specimen donors and research groups associated with tumor samples acquisition and analysis associated with the Cancer Genome Atlas Research Network (TCGA).

\footnotetext{
The project was funded by a National Health and Medical Research Council of Australia (NH\&MRC) Program Grant (1132519), Project Grant (1098960) and Development Grant (1093566), a Cancer Council of Queensland (CCQ) Project Grant (1083776), a Cancer Research Institute CLIP grant, and a research agreement with Bristol Myers Squibb. M. J. S. was supported by a Senior Principal Research Fellowship (1078671). M. W. L. T. was supported by a CDF1 Fellowship and NH\&MRC Project Grant (1098960). R. A. S. is supported by a NH\&MRC Practitioner Fellowship.
} 


\section{References}

1. Schachter J, Ribas A, Long GV, Arance A, Grob JJ, Mortier L, et al. Pembrolizumab versus ipilimumab for advanced melanoma: final overall survival results of a multicentre, randomised, open-label phase 3 study (KEYNOTE-006). Lancet 2017;390(10105):1853-62 doi 10.1016/ s0140-6736(17)31601-X. [PubMed: 28822576]

2. O’Donnell JS, Long GV, Scolyer RA, Teng MW, Smyth MJ. Resistance to PD1/PDL1 checkpoint inhibition. Cancer Treat Rev 2017;52:71-81 doi 10.1016/j.ctrv.2016.11.007. [PubMed: 27951441]

3. Dougall WC, Kurtulus S, Smyth MJ, Anderson AC. TIGIT and CD96: new checkpoint receptor targets for cancer immunotherapy. Immunol Rev 2017;276(1):112-20 doi 10.1111/imr.12518. [PubMed: 28258695]

4. Li XY, Das I, Lepletier A, Addala V, Bald T, Stannard K, et al. CD155 loss enhances tumor suppression via combined host and tumor-intrinsic mechanisms. J Clin Invest 2018;128(6):2613-25 doi 10.1172/JCI98769. [PubMed: 29757192]

5. Johnston RJ, Comps-Agrar L, Hackney J, Yu X, Huseni M, Yang Y, et al. The immunoreceptor TIGIT regulates antitumor and antiviral CD8(+) T cell effector function. Cancer Cell 2014;26(6): 923-37 doi 10.1016/j.ccell.2014.10.018. [PubMed: 25465800]

6. Kurtulus S, Sakuishi K, Ngiow SF, Joller N, Tan DJ, Teng MW, et al. TIGIT predominantly regulates the immune response via regulatory T cells. J Clin Invest 2015;125(11):4053-62 doi 10.1172/JCI81187. [PubMed: 26413872]

7. Blake SJ, Stannard K, Liu J, Allen S, Yong MC, Mittal D, et al. Suppression of Metastases Using a New Lymphocyte Checkpoint Target for Cancer Immunotherapy. Cancer Discov 2016;6(4):446-59 doi 10.1158/2159-8290.CD-15-0944. [PubMed: 26787820]

8. Roman Aguilera A, Lutzky VP, Mittal D, Li XY, Stannard K, Takeda K, et al. CD96 targeted antibodies need not block CD96-CD155 interactions to promote NK cell anti-metastatic activity. Oncoimmunology 2018;7(5):e1424677 doi 10.1080/2162402X.2018.1424677. [PubMed: 29721390]

9. Lepletier A, Lutzky VP, Mittal D, Stannard K, Watkins TS, Ratnatunga CN, et al. The immune checkpoint CD96 defines a distinct lymphocyte phenotype and is highly expressed on tumorinfiltrating T cells. Immunol Cell Biol 2018 doi 10.1111/imcb.12205.

10. Mittal D, Vijayan D, Putz EM, Aguilera AR, Markey KA, Straube J, et al. Interleukin-12 from CD103(+) Batf3-dependent dendritic cells required for NK-cell suppression of metastasis. Cancer Immunol Res 2017;5(12):1098-108 doi 10.1158/2326-6066.CIR-17-0341. [PubMed: 29070650]

11. Ahern E, Harjunpaa H, O’Donnell JS, Allen S, Dougall WC, Teng MWL, et al. RANKL blockade improves efficacy of PD1-PD-L1 blockade or dual PD1-PD-L1 and CTLA4 blockade in mouse models of cancer. Oncoimmunology 2018;7(6):e1431088 doi 10.1080/2162402x.2018.1431088. [PubMed: 29872559]

12. Gao Y, Souza-Fonseca-Guimaraes F, Bald T, Ng SS, Young A, Ngiow SF, et al. Tumor immunoevasion by the conversion of effector NK cells into type 1 innate lymphoid cells. Nat Immunol 2017;18(9):1004-15 doi 10.1038/ni.3800. [PubMed: 28759001]

13. Swann JB, Hayakawa Y, Zerafa N, Sheehan KC, Scott B, Schreiber RD, et al. Type I IFN contributes to NK cell homeostasis, activation, and antitumor function. J Immunol 2007;178(12): 7540-9. [PubMed: 17548588]

14. Tirosh I, Izar B, Prakadan SM, Wadsworth MH, 2nd, Treacy D, Trombetta JJ, et al. Dissecting the multicellular ecosystem of metastatic melanoma by single-cell RNA-seq. Science 2016;352(6282): 189-96 doi 10.1126/science.aad0501. [PubMed: 27124452]

15. Siemers NO, Holloway JL, Chang H, Chasalow SD, Ross-MacDonald PB, Voliva CF, et al. Genome-wide association analysis identifies genetic correlates of immune infiltrates in solid tumors. PLoS One 2017;12(7):e0179726 doi 10.1371/journal.pone.0179726. [PubMed: 28749946]

16. Spranger S, Dai D, Horton B, Gajewski TF. Tumor-residing Batf3 dendritic cells are required for effector T cell trafficking and adoptive T cell therapy. Cancer Cell 2017;31(5):711-23 e4 doi 10.1016/j.ccell.2017.04.003. [PubMed: 28486109] 
17. Zitvogel L, Kroemer G. CD103+ dendritic cells producing interleukin-12 in anticancer immunosurveillance. Cancer Cell 2014;26(5):591-3 doi 10.1016/j.ccell.2014.10.008. [PubMed: 25517740]

18. Salmon H, Idoyaga J, Rahman A, Leboeuf M, Remark R, Jordan S, et al. Expansion and activation of CD103(+) dendritic cell progenitors at the tumor site enhances tumor responses to therapeutic PD-L1 and BRAF inhibition. Immunity 2016;44(4):924-38 doi 10.1016/j.immuni.2016.03.012. [PubMed: 27096321]

19. Sanchez-Paulete AR, Cueto FJ, Martinez-Lopez M, Labiano S, Morales-Kastresana A, RodriguezRuiz ME, et al. Cancer immunotherapy with immunomodulatory anti-CD137 and anti-PD-1 monoclonal antibodies requires BATF3-dependent dendritic cells. Cancer Discov 2016;6(1):71-9 doi 10.1158/2159-8290.CD-15-0510. [PubMed: 26493961]

20. Le DT. Immunotherapy in colorectal cancer. Clin Adv Hematol Oncol 2018;16(1):22-4. [PubMed: 29741500]

21. Blake SJ, Dougall WC, Miles JJ, Teng MW, Smyth MJ. Molecular Pathways: Targeting CD96 and TIGIT for cancer immunotherapy. Clin Cancer Res 2016;22(21):5183-8 doi 10.1158/1078-0432.CCR-16-0933. [PubMed: 27620276]

22. Chauvin JM, Pagliano O, Fourcade J, Sun Z, Wang H, Sander C, et al. TIGIT and PD-1 impair tumor antigen-specific CD8(+) T cells in melanoma patients. J Clin Invest 2015;125(5):2046-58 doi 10.1172/jci80445. [PubMed: 25866972]

23. Guillerey C, Harjunpaa H, Carrie N, Kassem S, Teo T, Miles K, et al. TIGIT immune checkpoint blockade restores CD8(+) T cell immunity against multiple myeloma. Blood 2018 doi 10.1182/ blood-2018-01-825265.

24. Johnston RJ, Yu X, Grogan JL. The checkpoint inhibitor TIGIT limits antitumor and antiviral CD8(+) T cell responses. Oncoimmunology 2015;4(9):e1036214 doi 10.1080/2162402X. 2015.1036214. [PubMed: 26405604]

25. Cella M, Presti R, Vermi W, Lavender K, Turnbull E, Ochsenbauer-Jambor C, et al. Loss of DNAM-1 contributes to CD8+ T-cell exhaustion in chronic HIV-1 infection. Eur J Immunol 2010;40(4):949-54 doi 10.1002/eji.200940234. [PubMed: 20201043]

26. Guillerey C, Ferrari de Andrade L, Vuckovic S, Miles K, Ngiow SF, Yong MC, et al. Immunosurveillance and therapy of multiple myeloma are CD226 dependent. J Clin Invest 2015;125(5):2077-89 doi 10.1172/jci77181. [PubMed: 25893601]

27. Larkin J, Chiarion-Sileni V, Gonzalez R, Grob JJ, Cowey CL, Lao CD, et al. Combined nivolumab and ipilimumab or monotherapy in untreated melanoma. N Engl J Med 2015;373(1):23-34 doi 10.1056/NEJMoa1504030. [PubMed: 26027431]

28. Boutros C, Tarhini A, Routier E, Lambotte O, Ladurie FL, Carbonnel F, et al. Safety profiles of anti-CTLA-4 and anti-PD-1 antibodies alone and in combination. Nat Rev Clin Oncol 2016;13(8): 473-86 doi 10.1038/nrclinonc.2016.58. [PubMed: 27141885]

29. Harjunpaa H, Blake SJ, Ahern E, Allen S, Liu J, Yan J, et al. Deficiency of host CD96 and PD-1 or TIGIT enhances tumor immunity without significantly compromising immune homeostasis. Oncoimmunology 2018;7(7):e1445949 doi 10.1080/2162402X.2018.1445949. [PubMed: 29900061] 
A

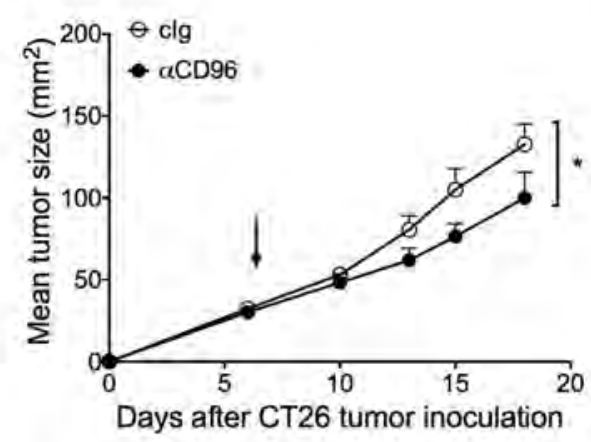

C

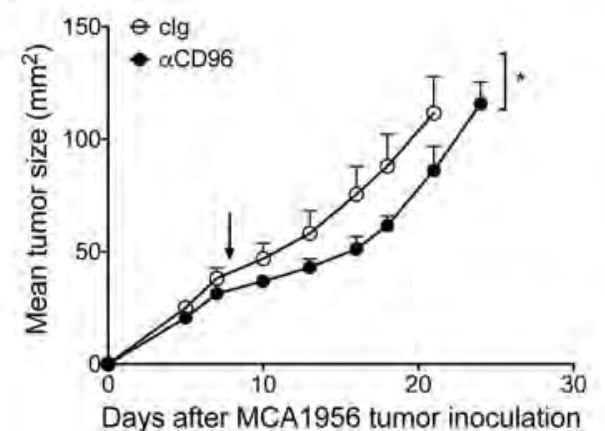

E

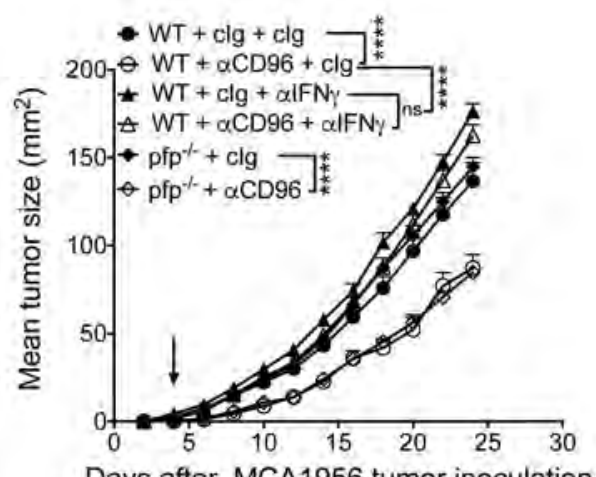

B

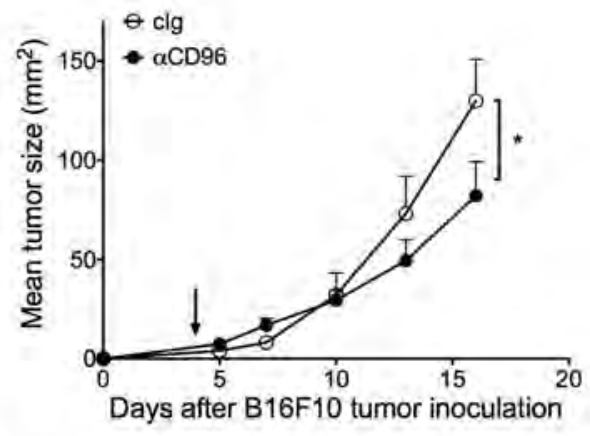

D

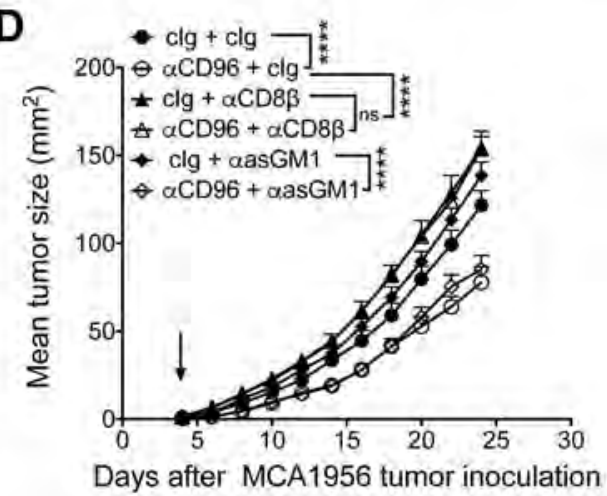

F

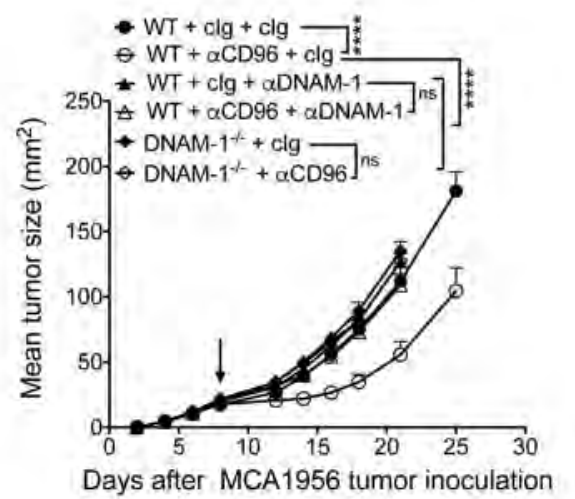

Figure 1. Anti-CD96 therapy efficacy requires $\mathrm{CD8}^{+} \mathrm{T}$ cells and IFN- $\gamma$.

(A) $2 \times 10^{5}$ CT26 colon carcinoma cells, (B) $1 \times 10^{5}$ B16F10 melanoma cells and C) $1 \times$ $10^{6}$ MCA1956 fibrosarcoma cells were injected subcutaneously in the BALB/c (A) or C57BL/6 WT mice (B, C) and $250 \mu \mathrm{g}$ of anti-CD96 (3.3) or control IgG (1-1) was injected i.p. on days 7, 10, 14 and 17 (A, C) or on days 3, 6, 10 and 13 (B). (D-F) $1 \times 10^{6}$ MCA1956 cells were injected subcutaneously in the C57BL/6 WT or gene targeted mice as indicated and $250 \mu \mathrm{g}$ of anti-CD96 (3.3) or control IgG (1-1) was injected i.p. on days 3, 7, 11, 15 (D, E) or on days 8, 12, 15 and 18 (F). (D) Additionally, some groups were injected i.p. on days 2, 3, 10 and 17 with anti-asialoGM1 $(50 \mu \mathrm{g})$ to deplete NK cells or anti-CD8 $\beta$ (53.5.8, 100 
$\mu \mathrm{g})$ to deplete $\mathrm{CD} 8^{+} \mathrm{T}$ cells. (E) Tumor cells were injected in WT or perforin gene targeted $\left(\mathrm{pfp}^{-/-}\right)$mice and neutralizing IFN $\gamma \mathrm{mAb}(\mathrm{H} 22,250 \mu \mathrm{g})$ was injected i.p. alone or in combination with anti-CD96 (3.3) on days 2, 310 and 17. (F) Tumor cells were injected in WT or DNAM-1 gene-targeted (DNAM-1 ${ }^{-/}$) mice and neutralizing DNAM-1 mAb (480.1, $250 \mu \mathrm{g}$ ) was injected i.p. alone or in combination with anti-CD96 (3.3) on days 7, 8, 15 and 21. Experiments A-F were performed once. Primary tumor growth was monitored by measuring two perpendicular diameters every 2-3 days. Means \pm SEM of $n=5-6$ mice/group is shown. Significant differences between groups were determined by Two-way ANOVA Turkey's multiple comparison test (*, P < 0.05; ****, P $<0.0001$; ns, not significant). For $1 \mathrm{C}$ and $1 \mathrm{~F}$, significant differences were shown for day 21 . Arrow indicates the day antiCD96 therapy was initiated. 

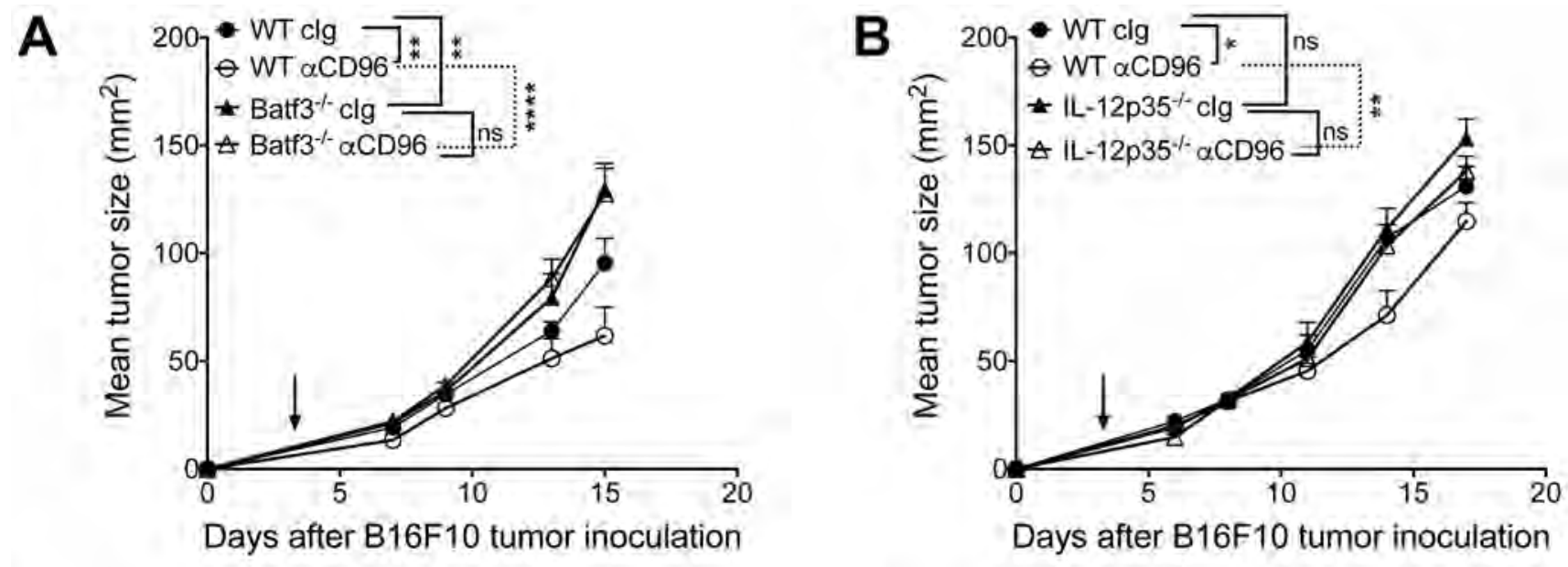

Days after B16F10 tumor inoculation
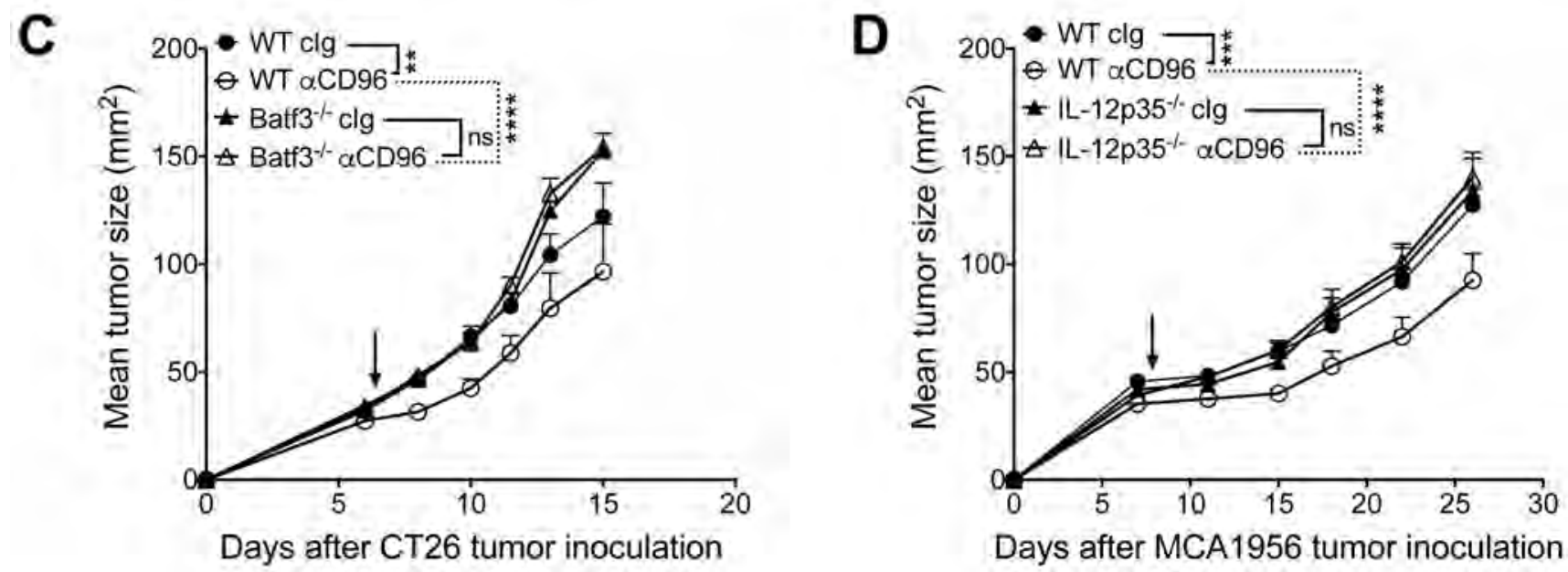

Figure 2. Anti-CD96 therapy is Batf3- and IL12p35-dependent.

(A) $1 \times 10^{5}$ B16F10 melanoma cells were injected subcutaneously into the (A) WT and Batf $^{-1-}$ or (B) WT and IL12p35 ${ }^{-/-}$mice. Anti-CD96 (3.3, $\left.250 \mu \mathrm{g} / \mathrm{mouse}\right)$ w as injected i.p. on day 3, 6, 10 and 13 . (C) $2 \times 10^{5}$ CT26 colon carcinoma cells were injected subcutaneously into the WT or Batf3 ${ }^{-/-}$mice. Anti-CD96 (3.3, $\left.250 \mu \mathrm{g} / \mathrm{mouse}\right)$ was injected i.p. on day 7, 10, 14 and 17. (D) $1 \times 10^{6}$ MCA1956 fibrosarcoma cells were injected subcutaneously into WT and IL12p35 ${ }^{-/-}$mice and anti-CD96 (3.3) or control IgG (1-1) was injected i.p. on days 8, 11, 15 and 18. Experiments A-D were performed once. Mice were monitored for tumor growth and means \pm SEM of $n=5-8$ mice/group are shown. Significant differences between groups were determined by two-way ANOVA Turkey's multiple comparison test $(*, \mathrm{P}<0.05$; **, $\mathrm{P}<0.01$; ***, $\mathrm{P}<0.001$; ****, $\mathrm{P}<0.0001$; ns, not significant). Arrow indicates the day anti-CD96 therapy was initiated. 
A
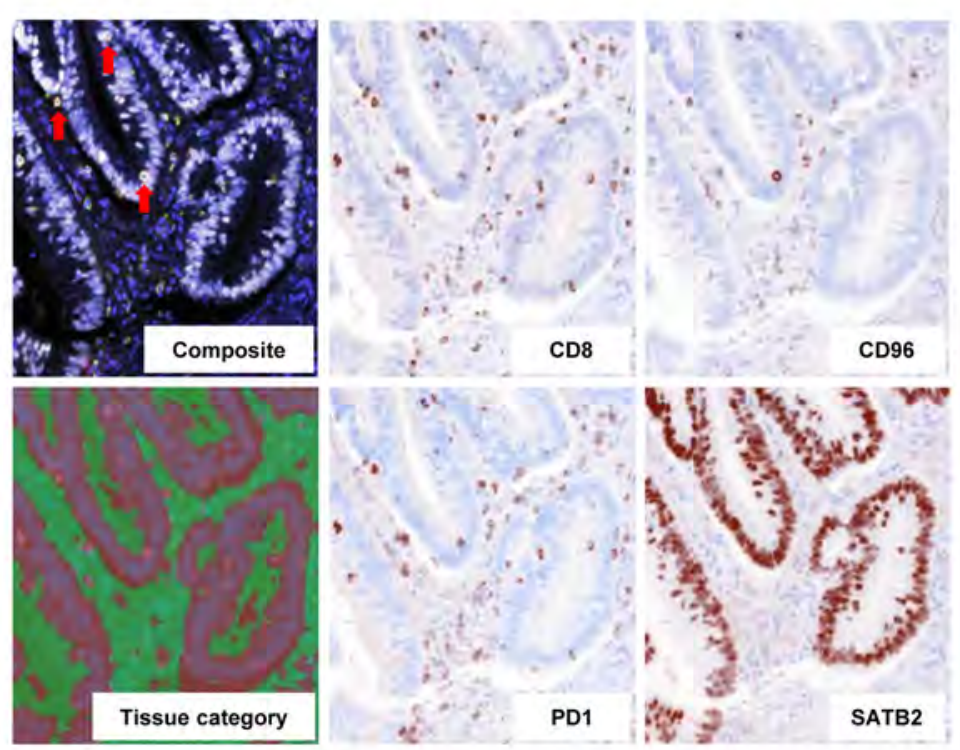

B
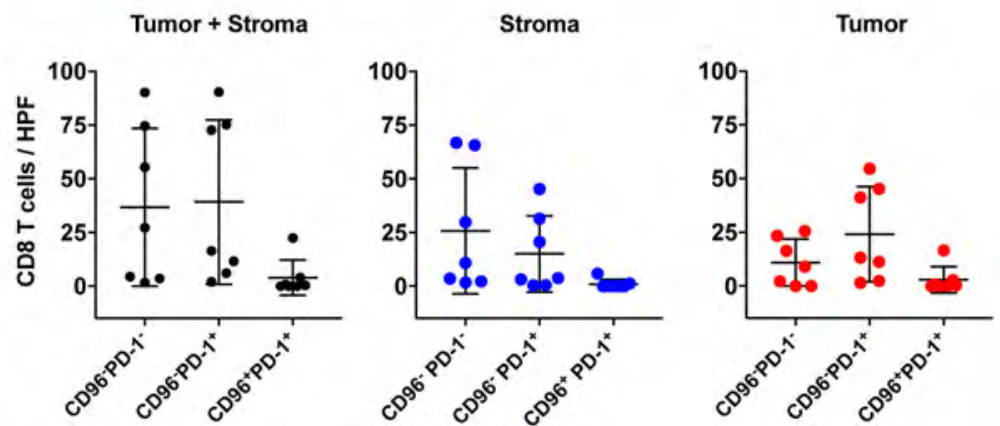

C
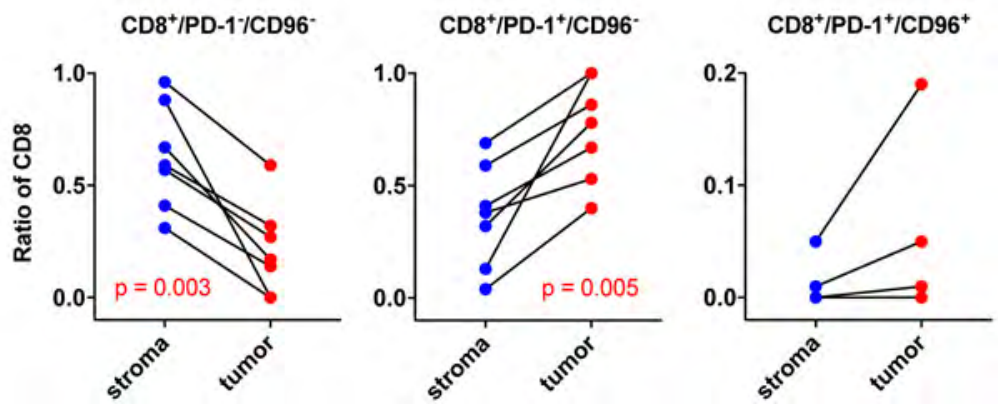

Figure 3. $\mathrm{CD96}^{+}$immune cell infiltration in microsatellite unstable colorectal cancers.

Expression of CD8, PD-1, and CD96 by tumor infiltrating lymphocytes was quantified using mIHF in a cohort of MSI-positive human primary colorectal cancers $(\mathrm{N}=7)$. SATB2 expression was used to segment tumors into tissue category areas; tumor parenchyma (tumor) or tumor stroma (stroma). Cell densities were recorded as average cell count per 20x high power field of view (cells/HPF). (A) Representative mIHF composite image of CRC with CD8 (green), PD-1 (red), CD96 (yellow), and SATB2 (white). Red arrows indicate triple colocalization of CD8/PD-1/CD96 on infiltrating lymphocytes in the tumor parenchyma. Tissue category map showing tumor regions (red) and stroma regions (green). 
Pseudo-pathology views representing single-plex expression for each marker (brown). (B) Average cell density per HPF is shown for each cell type by; total tumor area (black dots), stroma area (blue dots), tumor area (red dots). (C) Paired analysis showing the fraction of total $\mathrm{CD} 8^{+} \mathrm{T}$ cells of each cell type by tissue category area. Statistical analyses were performed using paired t-tests. 
A
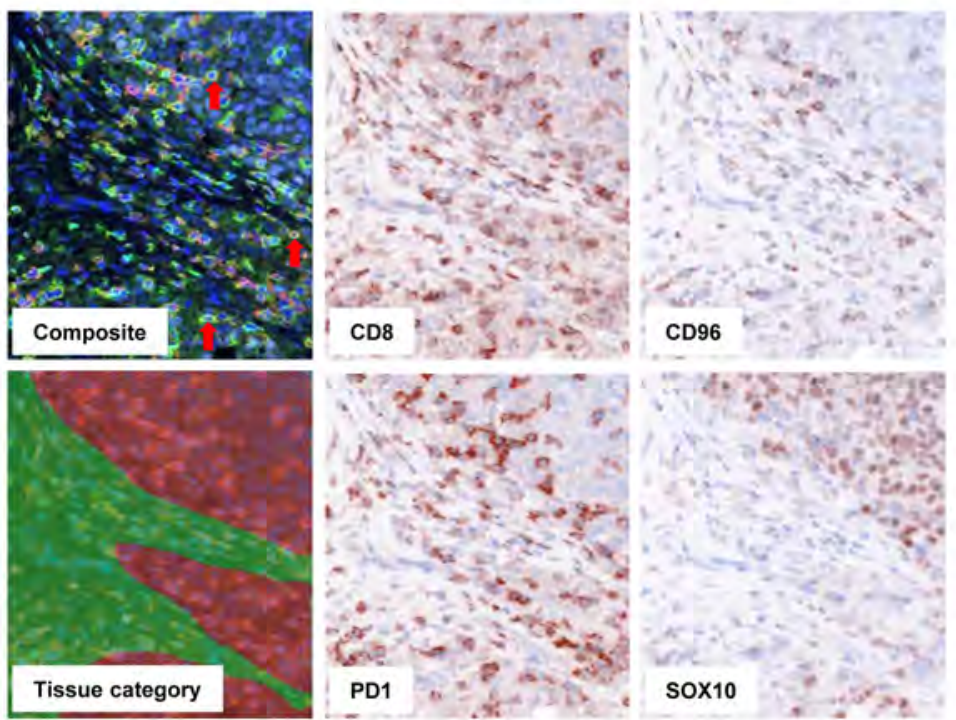

B
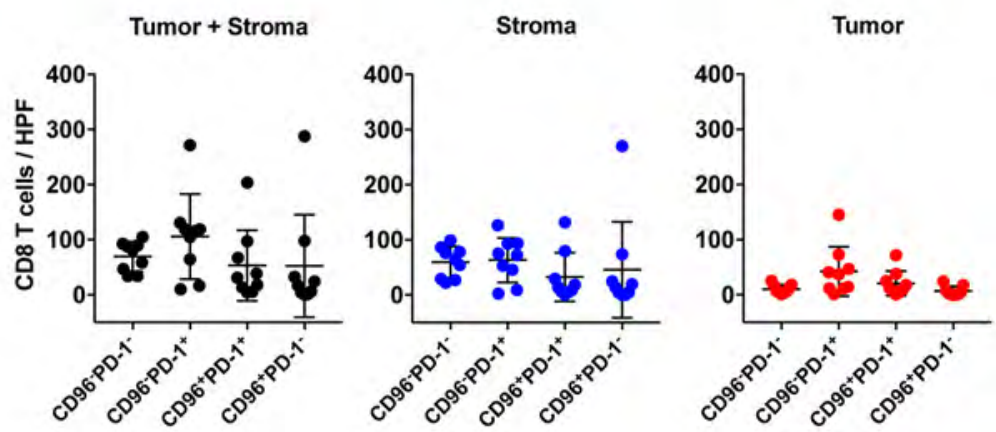

C
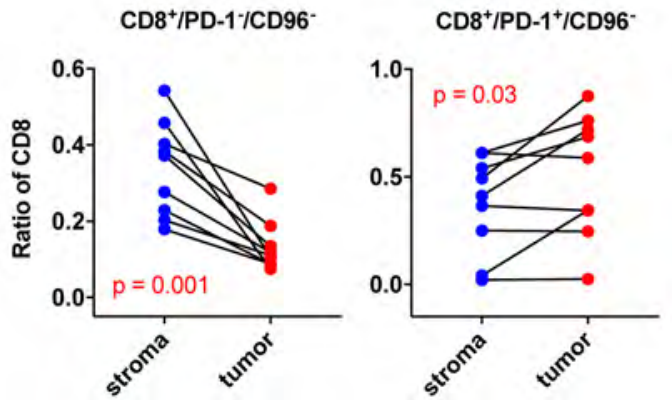

\section{CD8 $8^{+} / \mathrm{PD}-1^{+} / \mathrm{CD}^{+} 6^{+}$}

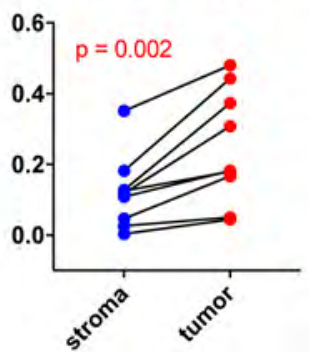

Figure 4. $\mathrm{CD96}^{+}$immune cell infiltration in metastatic melanoma.

Expression of CD8, PD-1, and CD96 by tumor-infiltrating lymphocytes was quantified using mIHF in a cohort of metastatic melanoma $(\mathrm{N}=9)$. SOX10 expression was used to segment tumors into tissue category areas; tumor parenchyma (tumor) or tumor stroma (stroma). Cell densities were recorded as average cell count per 20x high power field of view (cells/HPF). (A) Representative mIHF composite image of melanoma with CD8 (green), PD-1 (red), CD96 (yellow), and SOX10 (white). Red arrows indicate triple colocalization of CD8/PD-1/ CD96 on infiltrating lymphocytes in the tumor parenchyma. Tissue category map showing tumor regions (red) and stroma regions (green). Pseudo-pathology views representing 
singleplex expression for each marker (brown). (B) Average cell density per HPF is shown for each cell type by; total tumor area (black dots), stroma area (blue dots), tumor area (red dots). (C) Paired analysis showing the fraction of total $\mathrm{CD} 8^{+} \mathrm{T}$ cells of each cell type by tissue category area. Statistical analyses were performed using paired t-tests. 


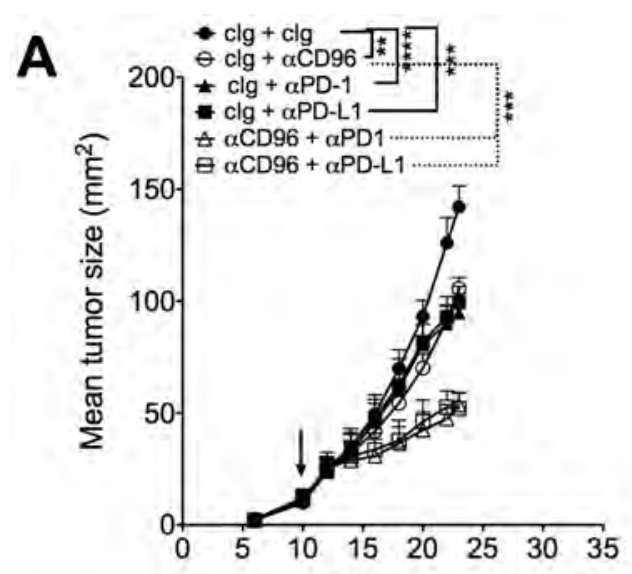

Days after CT26 tumor inoculation
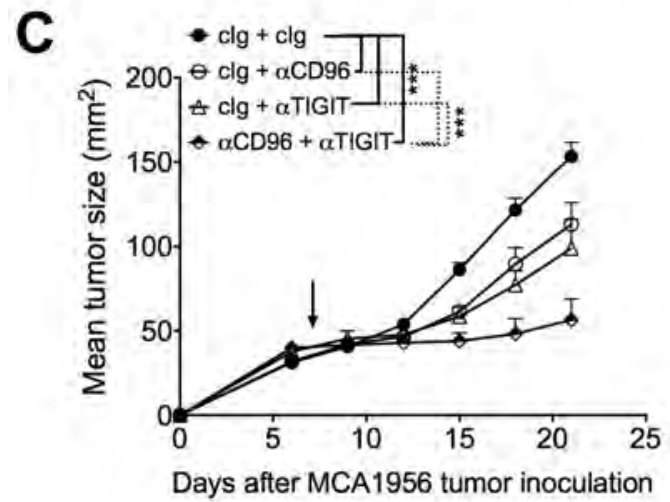

B

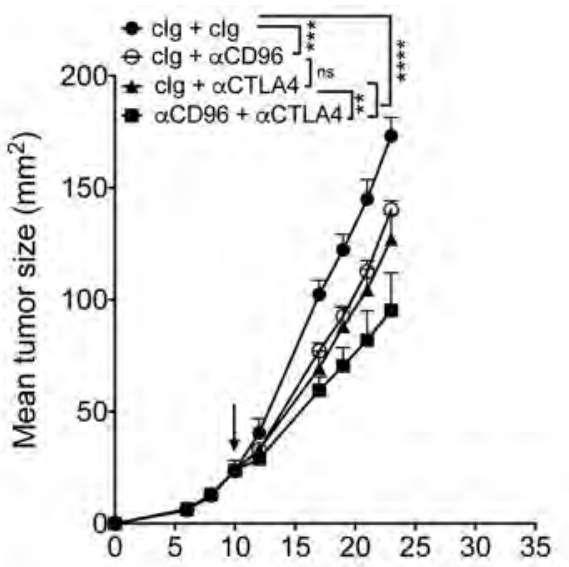

Days after CT26 tumor inoculation

D

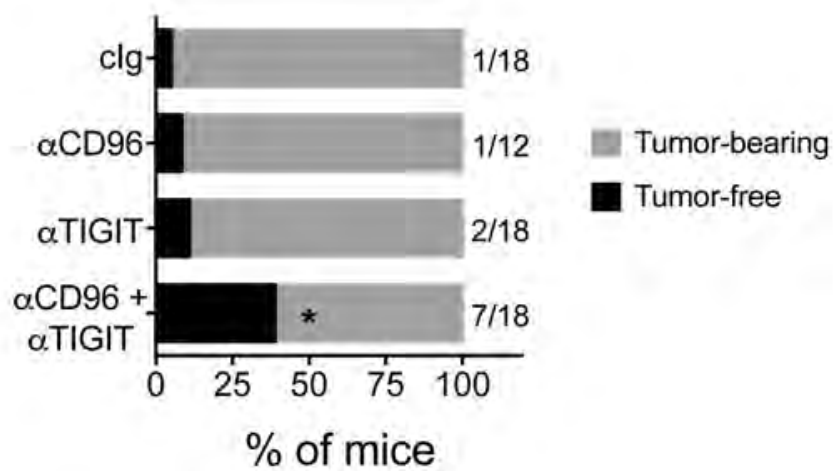

Figure 5. Anti-CD96 therapy combines with ICB to enhance suppression of primary tumor growth.

(A, B) $2 \times 10^{5}$ CT26 colon carcinoma cells were injected subcutaneously into the WT mice and anti-CD96 (3.3) or control IgG (1-1) was injected i.p. alone or in combination with i.p. anti-PD1 (250 $\mu \mathrm{g} / \mathrm{mouse})$ or anti-PD-L1 therapy (250 $\mu \mathrm{g} / \mathrm{mouse})(\mathrm{A})$ or with anti-CTLA-4 $\left(250 \mu \mathrm{g} /\right.$ mouse) (B) on days 10, 13, 17 and 20. (C, D) $1 \times 10^{6}$ MCA1956 fibrosarcoma cells were injected subcutaneously into the WT mice. Anti-CD96 (3.3, $250 \mu \mathrm{g})$ or control IgG (1-1) alone or in combination with anti-TIGIT (Rat IgG2a) $(250 \mu \mathrm{g})$ was injected i.p. on days 7, 10, 14 and 17. (D) Bar graph showing percentage of MCA1956 tumor bearing and tumor-free mice at day 30 after anti-CD96 and/or anti-TIGIT therapy. Ratio represents the number of tumor-free mice out of total number of mice with tumors. Data was pooled from three experiments representative of C. Experiments A and B were performed once. Mice were monitored for tumor growth and means \pm SEM of $n=5-10$ mice/group are shown. Significant differences between groups were determined by Two-way ANOVA Turkey's multiple comparison test for A-C and by Fisher-exact test for $\mathrm{D}(*, \mathrm{P}<0.05$; **, $\mathrm{P}<0.01$; $* * *, \mathrm{P}<0.001 ; * * * *, \mathrm{P}<0.0001 ; \mathrm{ns}$, not significant). Arrow indicates the day immunotherapy was initiated. 

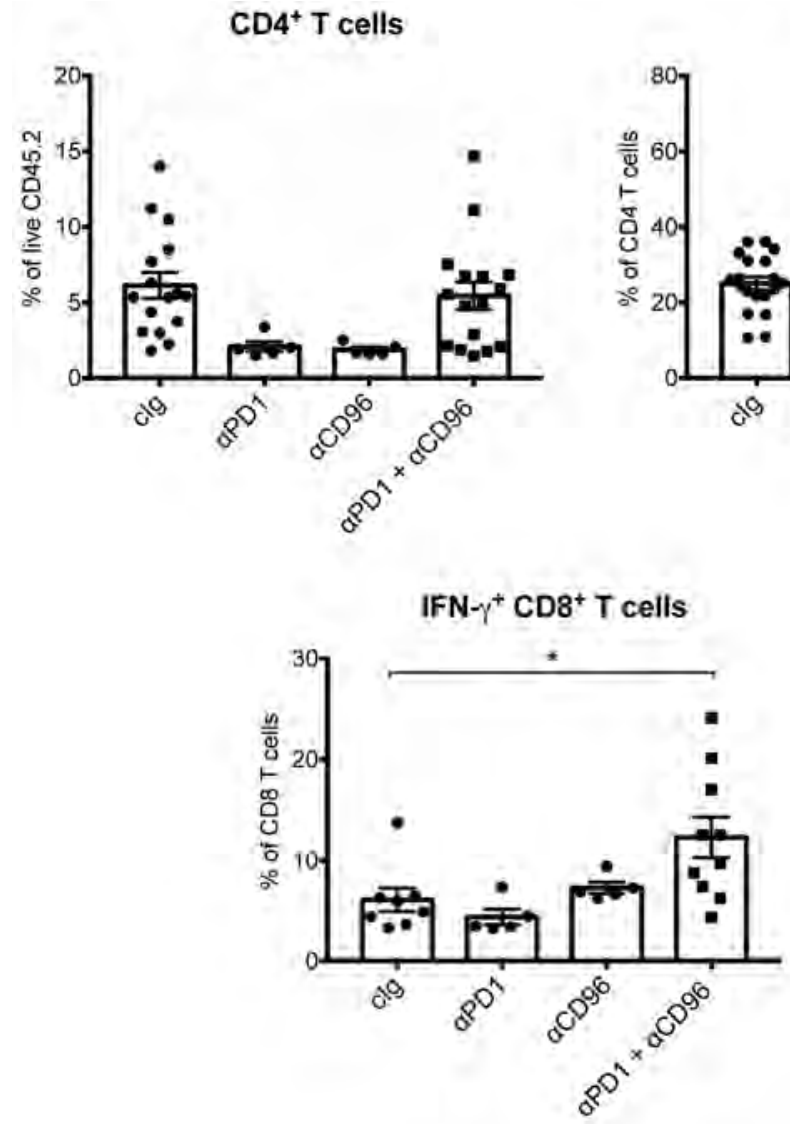

Tregs (CD4)

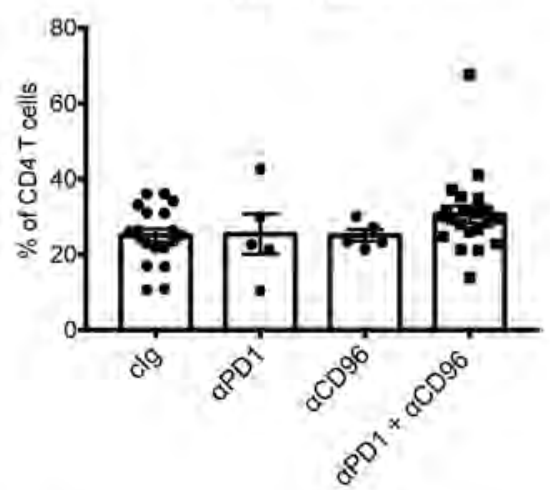

$\mathrm{CDB}^{+} \mathrm{T}$ cells

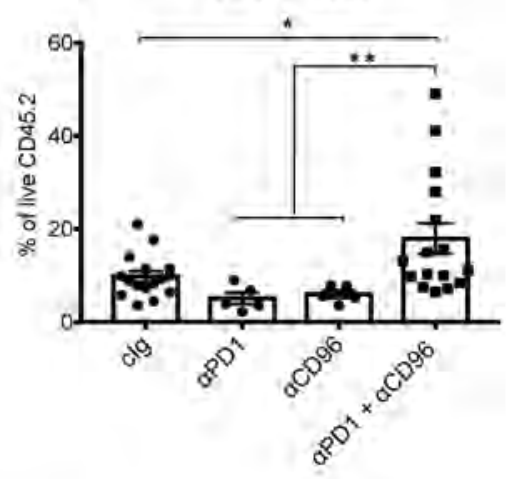

IFN $-\gamma^{+} \mathrm{TNF}^{+} \mathrm{CD}^{+} \mathrm{T}$ cells

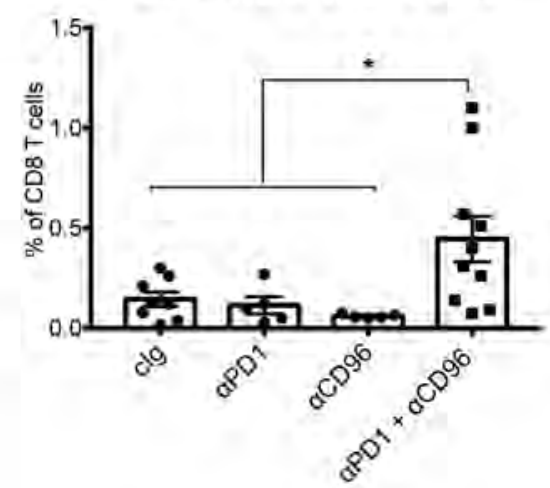

Figure 6. Combination of anti-CD96 with anti-PD1 therapy increases expression of IFN $\gamma$ in CD8 $^{+} \mathbf{T}$ cells.

$2 \times 10^{5}$ CT26 colon carcinoma cells were injected subcutaneously into the WT mice and tumors were treated or not with control Ig, anti-CD96, anti-PD1 or the combination of antiPD1 and anti-CD96 at day 8 and 11 and tumors were collected at day 14 for TILs analysis. Bar graph showing the percentage of total $\mathrm{CD}^{+}{ }^{+} \mathrm{T}$ cells, FoxP3 ${ }^{+}$regulatory $\mathrm{T}$ cells (Tregs) and $\mathrm{CD}^{+} \mathrm{T}$ cells, IFN- $\gamma$ expressing CD8 ${ }^{+} \mathrm{T}$ cells and IFN- $\gamma^{+} \mathrm{TNF}_{-\mathrm{a}^{+}}$expressing $\mathrm{CD} 8^{+} \mathrm{T}$ cells in the tumors treated or not with anti-CD96 and anti-PD1. Significant differences between groups were determined by Mann-Whitney $\mathrm{U}$ test $(*, \mathrm{P}<0.05$; **, $\mathrm{P}<0.01)$. Data was pooled from 1-3 independent experiments. 
A

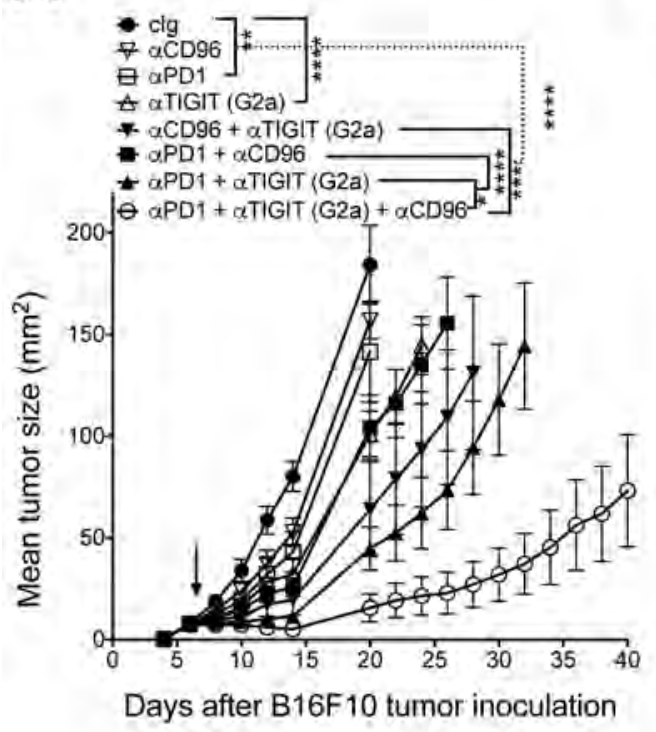

C

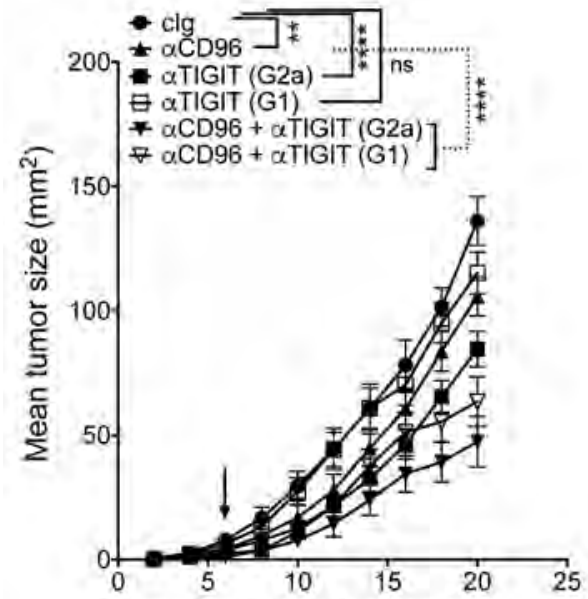

Days after B16F10 tumor inoculation
B

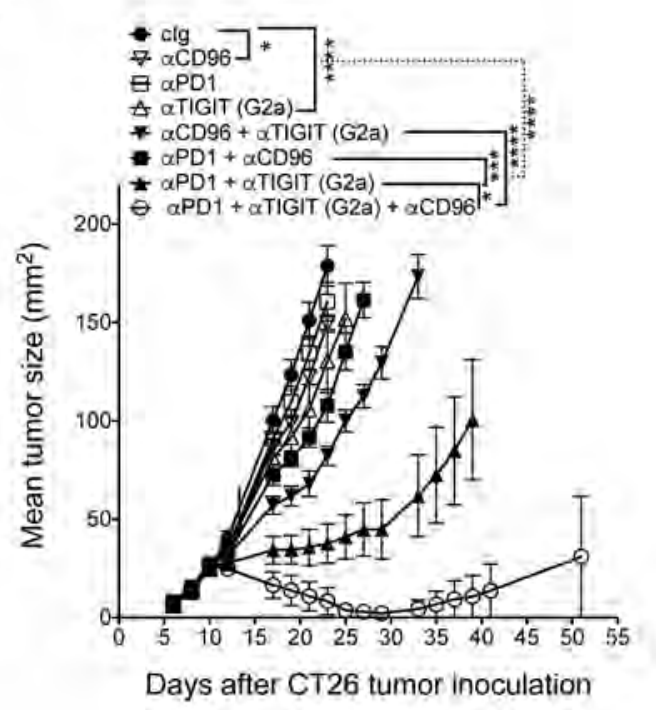

D

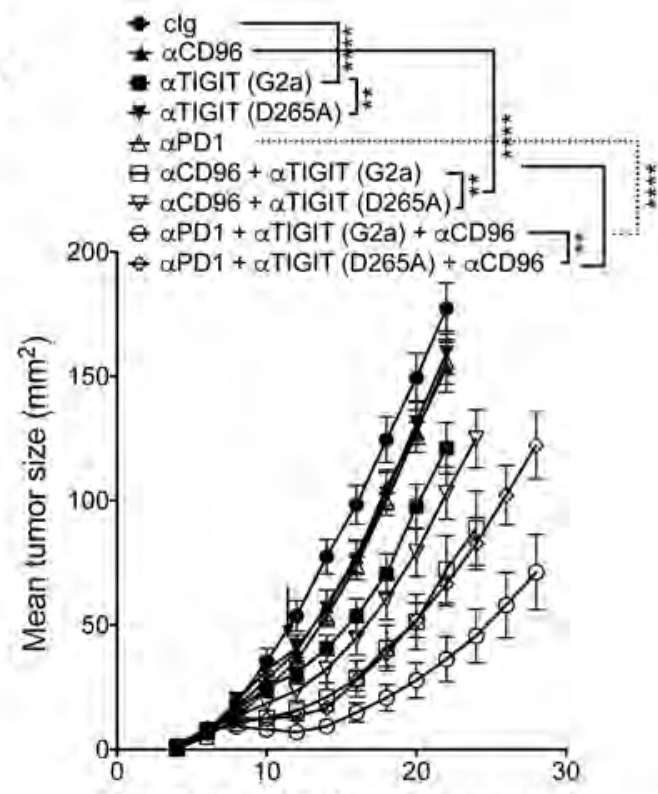

Days after B16F10 tumor inoculation

Figure 7. Anti-TIGIT mouse IgG2a enhances the therapeutic activity of CD96 and PD-1 blockade in triple combination therapy.

(A, B) $1 \times 10^{5}$ B16F10 melanoma cells (A) and $2 \times 10^{5}$ CT26 colon carcinoma cells (B) were injected subcutaneously into the WT mice. Anti-CD96 (3.3, $250 \mu \mathrm{g} / \mathrm{mouse})$, anti-PD1 (RMP1-14, $250 \mu \mathrm{g} / \mathrm{mouse}$ ) and/or anti-TIGIT (4B1, $250 \mu \mathrm{g} / \mathrm{mouse}$ ) were injected i.p. on days 6, 9, 12 and 15 (A) or on days 10,13, 17 and 20 (B) and tumor growth was monitored. (C, D) Same as A, except that TIGIT antibodies (4B1, $250 \mu \mathrm{g} / \mathrm{mouse}$ ) with two different IgG backgrounds (G2a vs G1) in C and Fc active IgG2a (G2a) vs Fc mutant (D265A) in (D) were used for experiments. Experiments A-D were performed once. Mice were monitored 
for tumor growth and means \pm SEM of $n=5-10$ mice/group are shown. Significant differences between groups were determined by Two-way ANOVA Tukey's multiple comparison test $(*, \mathrm{P}<0.05 ; * *, \mathrm{P}<0.01 ; * * *, \mathrm{P}<0.001 ; * * * *, \mathrm{P}<0.0001 ; \mathrm{ns}$, not significant). Significant differences between groups are shown at day 20 (A and C), day 23 (B) and day 22 (D). Arrow indicates the day immunotherapy was initiated. 

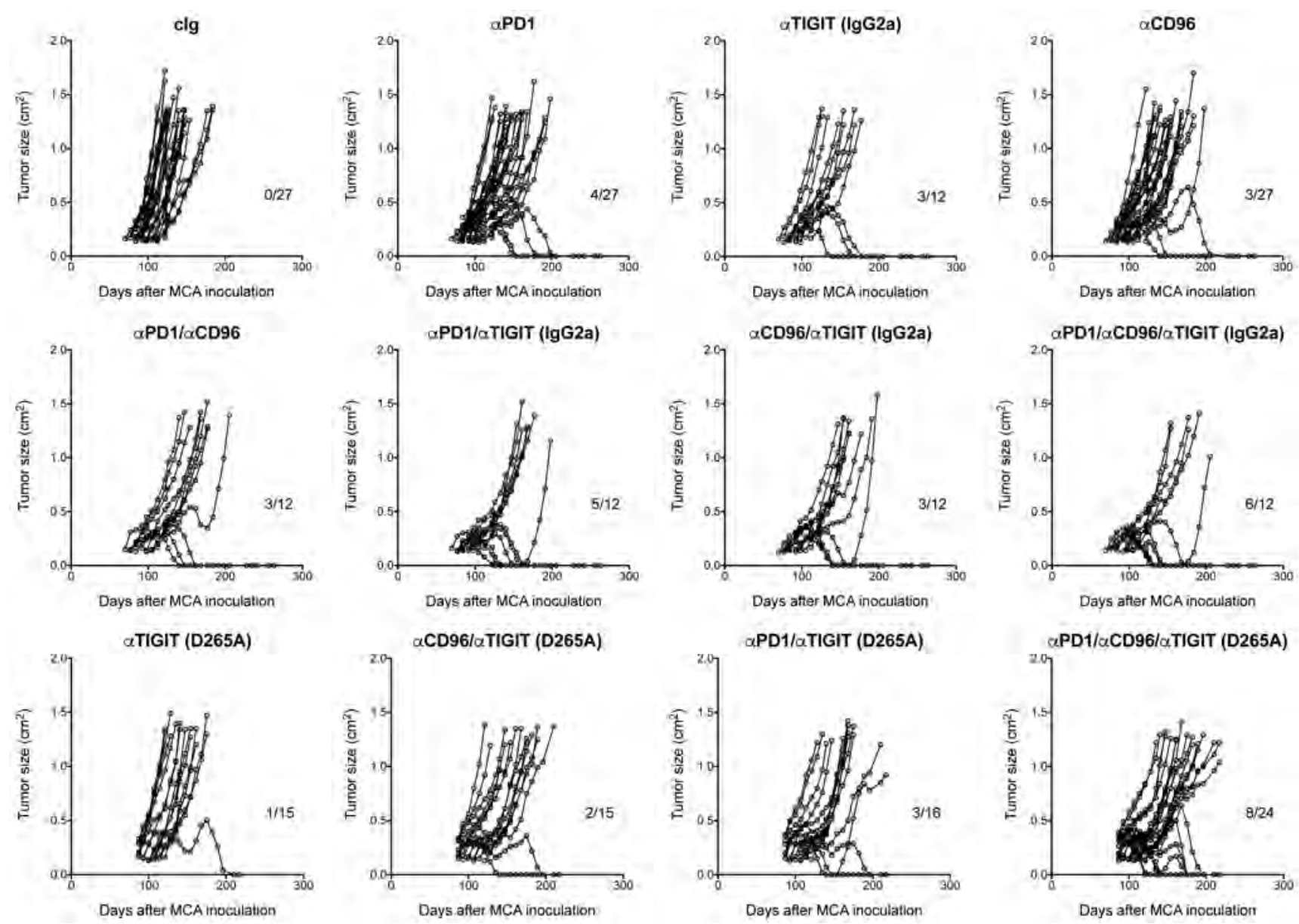

Figure 8. The combination of anti-CD96 with anti-PD1 and/or anti-TIGIT therapy inhibits the growth of established de novo fibrosarcoma tumors.

Groups of 12-15 male C57BL/6 WT mice were inoculated s.c. in the hind flank with $300 \mu \mathrm{g}$ of MCA in $0.1 \mathrm{~mL}$ of corn oil as described in the Materials and Methods. Mice were treated with cIg (1-1), anti-CD96 (3.3), anti-PD-1 (RMP1-14), anti-TIGIT (4B1, IgG2a), antiTIGIT (4B1, D265A) or their combinations (100 $\mu$ g each i.p., twice/week) for 6 weeks from the second palpable tumor measurement $\left(0.1-0.4 \mathrm{~cm}^{2}\right.$, days $84-147$ relative to MCA inoculation). Mice were then monitored for fibrosarcoma development for 250 days with measurements made with a caliper as the product of two perpendicular diameters $\left(\mathrm{cm}^{2}\right)$. Results are a pool of two experiments. Number of mice that rejected tumors out of total number of mice is shown. 